\title{
ENDOTRIVIAL MODULES FOR THE GENERAL LINEAR GROUP IN A NONDEFINING CHARACTERISTIC
}

\author{
JON F. CARLSON, NADIA MAZZA, AND DANIEL K. NAKANO
}

\begin{abstract}
Suppose that $G$ is a finite group such that $\operatorname{SL}(n, q) \subseteq G \subseteq \operatorname{GL}(n, q)$, and that $Z$ is a central subgroup of $G$. Let $T(G / Z)$ be the abelian group of equivalence classes of endotrivial $k(G / Z)$-modules, where $k$ is an algebraically closed field of characteristic $p$ not dividing $q$. We show that the torsion free rank of $T(G / Z)$ is at most one, and we determine $T(G / Z)$ in the case that the Sylow $p$-subgroup of $G$ is abelian and nontrivial. The proofs for the torsion subgroup of $T(G / Z)$ use the theory of Young modules for $\operatorname{GL}(n, q)$ and a new method due to Balmer for computing the kernel of restrictions in the group of endotrivial modules.
\end{abstract}

\section{INTRODUCTION}

For a finite group $G$, endotrivial modules form an important part of the Picard group of self-equivalences of the stable module category, $\operatorname{stmod}(k G)$, for the group algebra $k G$. In particular, the functor of tensoring with an endotrivial module is a self-equivalence of "Morita type" on the stable category as well as on the derived category. The isomorphism classes in the stable category of the endotrivial modules over $G$ form a group, $T(G)$, under tensor product. The endotrivial group $T(G)$ was introduced by Dade $[14,15]$ who showed that $T(G)$ is cyclic in the case that $G$ is an abelian $p$-group. For general $p$-groups a classification of the endotrivial modules was obtained by the first author and Thévenaz $[11,12,6]$, building on work of Alperin [1] and others.

The problem of computing $T(G)$ for an arbitrary finite group $G$ remains open. Many results for specific groups can be found in $[7,8,9,10,19,21,22]$. It is hoped that a complete solution to calculating $T(G)$ can be reduced to almost-simple groups. The proof in [10] hints that such a reduction may hold. The fact that $T(G)$ is finitely generated can be derived from the theory of Green correspondents and the classification of endotrivial modules over $p$-groups.

This paper is part of a series of efforts to classify endotrivial modules for almost simple groups. In [8], the authors gave a classification of the endotrivial modules for the finite groups of Lie type where $p$ is the defining characteristic. Here, we consider the case of finite groups of Lie type in nondefining characteristics. We prove that the torsion free part of the group of endotrivial modules has either rank zero or one if the group has underlying root system of type $A_{n}$, and we characterize the torsion

Date: May 28, 2014.

Research of the first author was supported in part by NSF grant DMS-1001102.

Research of the third author was supported in part by NSF grant DMS-1002135. 
part of the group provided the Sylow $p$-subgroup is abelian. The main results are the following. For notation, let $k$ be an algebraically closed field of characteristic $p>0$ with $\operatorname{gcd}(p, q)=1$.

First we present the case when the Sylow $p$-subgroup is not cyclic, which is by far more difficult than when the Sylow $p$-subgroup is cyclic.

Theorem 1.1. Suppose that $\operatorname{SL}(n, q) \subseteq G \subseteq \operatorname{GL}(n, q)$ and that $Z \subseteq Z(G)$. Assume that $p$ divides the order of $G$ and that the Sylow p-subgroup of $G$ is abelian but not cyclic. Then

$$
T(G / Z) \cong \mathbb{Z} \oplus X(G / Z),
$$

where the torsion free part is generated by the class of $\Omega(k)$. Here $X(G / Z)$ is the group of isomorphism classes of $k(G / Z)$-modules of dimension one.

An interesting feature of the proof of this theorem is the first time utilization of a powerful method recently introduced by Balmer [3]. If $H$ is a subgroup of $G$ that contains a Sylow $p$-subgroup of $G$, then Balmer's method finds the kernel of the restriction map $T(G) \rightarrow T(H)$ in combinatorial terms. Applying the method requires detailed information about the $H$ - $H$ double cosets in $G$ and the normalizers all of the $p$-subgroups of $G$. This makes the applications difficult except in some special cases.

For the sake of completion, we present also the case that the Sylow $p$-subgroup is cyclic. The proof in this case is accomplished by straightforward applications of known results.

Theorem 1.2. Suppose that $\mathrm{SL}(n, q) \subseteq G \subseteq \mathrm{GL}(n, q)$ and that $Z \subseteq Z(G)$. Assume that the Sylow p-subgroup $S$ of $G$ is cyclic and let $N=N_{G}(S)$. Then $T(G / Z) \cong$ $T(\widehat{N})$ where $\widehat{N}=N_{G / Z}(\widehat{S})$ and $\widehat{S}$ is a Sylow p-subgroup of $G / Z$. Moreover, $T(\widehat{N})$ is the middle term of a not necessarily split extension

$$
1 \longrightarrow X(\widehat{N}) \longrightarrow T(\widehat{N}) \longrightarrow T(\widehat{S}) \longrightarrow 0
$$

where $X(\widehat{N}) \cong N /(Z[N, N])$ is the group of isomorphism classes of $k \widehat{N}$-modules of dimension one. Let $D=\operatorname{Det}(G) \cong G / \operatorname{SL}(n, q)$ and let $d=|D|$. In the case that $Z=\{1\}$ we have the following.

(a) If $p=2$ then $n=1$, and $T(G) \cong D / S$.

(b) Suppose that $p>2$ divides $q-1$. If $p$ divides $d$, then $n=1$ and $T(G) \cong$ $\mathbb{Z} / a \mathbb{Z} \oplus \mathbb{Z} / 2 \mathbb{Z}$, where $d=a p^{t}$ for a relatively prime to $p$.

(c) If $p>2$ divides $q-1$ and $p$ does not divide $d$, then there are two possibilities:

(i) assuming that 2 does not divide $(q-1) / d$, then $T(G) \cong \mathbb{Z} / d \mathbb{Z} \oplus \mathbb{Z} / 4 \mathbb{Z}$.

(ii) assuming that 2 divides $(q-1) / d$, then $T(G) \cong \mathbb{Z} / d \mathbb{Z} \oplus \mathbb{Z} / 4 \mathbb{Z} \oplus \mathbb{Z} / 2 \mathbb{Z}$.

(d) Suppose that $p$ does not divide $q-1$. Let e be the least integer such that $p$ divides $q^{e}-1$. Then $n=e+f$ for some $f$ with $0 \leq f<e$. Let $m=(q-1) / d$ and $\ell=\operatorname{gcd}\left(m(q-1), q^{e}-1\right) / m$. Then we have two possibilities:

(i) if $f=0$ then $T(G) \cong \mathbb{Z} / \ell \mathbb{Z} \oplus \mathbb{Z} / 2 e \mathbb{Z}$,

(ii) while if $f>0$, then $T(G) \cong \mathbb{Z} / 2 e \mathbb{Z} \oplus \mathbb{Z} /(q-1) \mathbb{Z} \oplus \mathbb{Z} / d \mathbb{Z}$ (except that $T(G) \cong \mathbb{Z} / 2 e \mathbb{Z} \oplus \mathbb{Z} / 2 \mathbb{Z}$ if both $f=2$ and $q=2)$. 
The paper is organized as follows. In Sections 2 and 3 of the paper we present some preliminary results on endotrivial modules and general linear groups. In particular, we prove that the torsion free rank of the group of endotrivial modules is never more than one. This is a consequence of known facts about the conjugations of $p$ subgroups in the general linear group. In Section 4, a proof is given for Theorem 1.1 in the case that $G=\operatorname{SL}(n, q)$ and $p$ divides $q-1$. Balmer's characterization of the kernel of restriction is reviewed in Section 5. In Section 6 notation and preliminaries are set up for Section 7 where an important case of Theorem 1.1 is proved. The remaining cases, for $G=\operatorname{SL}(n, q)$ with noncyclic Sylow $p$-subgroup, are considered in Sections 8 and 9. An essential feature of the proofs is the use of the theory of Young modules for the general linear group. In the last section, the results are assembled and the proofs of Theorems 1.1 and 1.2 are presented.

Acknowledgements: The authors would like to thank Paul Balmer for many helpful conversations and for urging us to look closely at his papers on the kernel of restriction. The second author acknowledges the Department of Mathematics at the University of Georgia for its support and hospitality during the initial stages of this project. The authors of this paper drew a lot of inspiration from experimental calculations using the computer algebra system Magma [5], though none of it is actually used in the proofs of this paper. We are grateful to Caroline Lassueur for pointing out an error in an earlier version of the paper. Finally, we wish to thank the referee for many helpful suggestions.

\section{Preliminaries}

Throughout this paper, let $k$ denote an algebraically closed field of prime characteristic $p$. We assume that all modules are finitely generated. If $A$ and $B$ are $k G$-modules for $G$ a finite group, we write $A \cong B \oplus$ (proj) to mean that $A$ is isomorphic to the direct sum of $B$ with some projective module. We write $k$ for the trivial $k G$-module. Unless otherwise specified, the symbol $\otimes$ is the tensor product $\otimes_{k}$ of the underlying vector spaces, and in case of $k G$-modules, this is a $k G$-module with $G$ acting via the diagonal action on the factors.

We start by reviewing some basics of the study of endotrivial modules and set some notation. If $M$ is a $k G$-module, and $\varphi: Q \rightarrow M$ its projective cover, then we let $\Omega^{1}(M)$, or simply $\Omega(M)$, denote the kernel of $\varphi$ (called the first syzygy of $M$ ). Likewise, if $\vartheta: M \rightarrow Q$ is the injective hull of $M$ (recall that $k G$ is a self-injective ring so $Q$ is also projective), then $\Omega^{-1}(M)$ denotes the cokernel of $\vartheta$. Inductively, we set $\Omega^{n}(M)=\Omega\left(\Omega^{n-1}(M)\right)$ and $\Omega^{-n}(M)=\Omega^{-1}\left(\Omega^{-n+1}(M)\right)$ for all integers $n>1$.

Assume that $G$ has order divisible by $p$. A $k G$-module $M$ is endotrivial provided its endomorphism algebra $\operatorname{End}_{k}(M)$ is isomorphic (as a $k G$-module) to the direct sum of the trivial module $k$ and a projective $k G$-module. Recall that $\operatorname{Hom}_{k}(M, N) \cong$ $M^{*} \otimes N$ where $M^{*}$ denotes the $k$-dual $\operatorname{Hom}_{k}(M, k)$ of $M$. In other words, a $k G$ module $M$ is endotrivial if and only if $\operatorname{Hom}_{k}(M, M) \cong M^{*} \otimes M \cong k \oplus$ (proj),

Any endotrivial module $M$ splits as the direct sum $M_{\diamond} \oplus$ (proj) for an indecomposable endotrivial $k G$-module $M_{\diamond}$, which is unique up to isomorphism. We define 
an equivalence relation

$$
M \sim N \Longleftrightarrow M_{\diamond} \cong N_{\diamond}
$$

on the class of endotrivial $k G$-modules, and let $T(G)$ be the set of equivalence classes. Every equivalence class contains a unique indecomposable module up to isomorphism. The tensor product induces an abelian group structure on the set $T(G)$ as in $[M]+[N]=[M \otimes N]$. The zero element of $T(G)$ is the class $[k]$ of the trivial module, consisting of all modules of the form $k \oplus$ (proj). The inverse of the class of a module $M$ is the class of the dual module $M^{*}$.

The group $T(G)$ is called the group of endotrivial $k G$-modules. It is known to be a finitely generated abelian group. In particular, the torsion subgroup $T T(G)$ of $T(G)$ is finite. We define $T F(G)=T(G) / T T(G)$. Thus, determining the structure of $T(G)$ can be split into two tasks: (i) compute $T F(G)$ and (ii) determine $T T(G)$.

The rank of the free abelian group $T F(G)$ is called the torsion-free rank of $T(G)$. It can be computed from a formula given in [8], which extends work of Alperin [1], and solely depends on the $p$-subgroup structure of $G$. Of particular interest is the $p$-rank of $G$ which is the rank of the largest elementary abelian $p$-subgroup of $G$ (i.e., the logarithm to base $p$ of the order of such subgroup). If the $p$-rank of $G$ is one, then $T F(G)=\{0\}$. For an elementary abelian $p$-group $E$ of rank at least 2, Dade $[14,15]$ proved that $T(E) \cong \mathbb{Z}$, and is generated by the class $[\Omega(k)]$. Now let $n_{G}$ be the number of conjugacy classes of maximal elementary abelian $p$-subgroups of $G$ of order $p^{2}$. These are maximal in the sense that they are not contained in a larger elementary abelian $p$-subgroup.

Theorem 2.1. ([8], Theorem 3.1) Assume that the p-rank of $G$ is at least 2. The torsion-free rank of $T(G)$ is equal to the number $n_{G}$ defined above if $G$ has p-rank 2 , and is equal to $n_{G}+1$ if $G$ has rank at least 3 .

In [12], it is shown that if $G$ is a $p$-group, then $T T(G)$ is trivial unless $G$ is cyclic, dihedral, generalized quaternion or semi-dihedral. In many cases, the subgroup $T T(G)$ is determined by the kernel of the restriction $T(G) \rightarrow T(S)$ where $S$ is a Sylow $p$-subgroup of $G$. We introduce the following term for the convenience of stating some of the results.

Definition 2.2. We say that a $k G$-module $M$ has trivial Sylow restriction, if the restriction of $M$ to a Sylow p-subgroup $S$ of $G$ has the form $M_{\downarrow S} \cong k \oplus$ (proj). Any such module is endotrivial and is the direct sum of an indecomposable trivial source module and a projective module.

The following is a straightforward application of Mackey formula (see Lemma 2.6 in $[20])$.

Proposition 2.3. Let $S$ be a Sylow p-subgroup of $G$. If $G$ has a nontrivial normal $p$-subgroup then every indecomposable $k G$-module with trivial Sylow restriction has dimension one.

Corollary 2.4. Suppose that $G=\mathrm{GL}(n, q)$ and $p$ divides $q-1$. Then every indecomposable $k G$-module with trivial Sylow restriction has dimension one. 
Proof. With these hypotheses, the center of $G$ has order divisible by $p$ and so $G$ has a nontrivial normal $p$-subgroup. The result now follows from Proposition 2.3.

Corollary 2.5. Suppose that $G=\operatorname{SL}(n, q)$ and $p$ divides both $q-1$ and $n$. If $M$ is an indecomposable endotrivial module with trivial Sylow restriction then $M$ is trivial.

Proof. In this case, the center of $G$ contains the scalar matrix $\zeta I_{n}$, where $I_{n}$ is the $n \times n$ identity matrix and $\zeta$ is a primitive $p^{t h}$ root of unity. Thus by Proposition $2.3, M$ has dimension one. Now observe that the only $k G$-module of dimension one is the trivial module since $\mathrm{SL}(n, q)$ is a perfect group.

Proposition 2.6. Suppose that $H \subseteq J$ are subgroups of $G$ such that $H$ contains a Sylow p-subgroup of $J$. Assume that there is a p-subgroup $Q$ of $H$, with the property that every left coset of $H$ in $J$ is represented by an element in the normalizer of $Q$. That is, assume that $J=N_{J}(Q) \cdot H$. If $M$ is an endotrivial $k J$-module such that $M_{\downarrow H} \cong X \oplus$ (proj) where $X$ has dimension one, then $M$ has dimension one. In particular, if every indecomposable endotrivial $k H$-module with trivial Sylow restriction has dimension one, then every indecomposable endotrivial $k J$-module with trivial Sylow restriction has dimension one.

Proof. Since $H$ contains a Sylow $p$-subgroup of $J$ it follows that $M$ is a direct summand of $X^{\uparrow J}$. By hypothesis, $Q$ acts trivially on $X$ and also trivially on

$$
X^{\uparrow J} \cong k J \otimes_{H} X \cong \sum_{x \in[J / H]} x \otimes X .
$$

That is, $\left(X^{\uparrow J}\right)_{\downarrow Q}$ is a direct sum of copies of $k_{Q}$, while $M_{\downarrow Q} \cong k_{Q} \oplus$ (proj). Hence, $M$ has dimension one.

We end this section with a description of the structure of the group of endotrivial modules in the case that a Sylow $p$-subgroup of $G$ is cyclic. The main point is that the subgroup $N$ in the following theorem has the property $N \cap{ }^{g} N$ is a $p^{\prime}$-group for any $g \notin N$. So the induction and restriction functors define equivalences between the stable categories of $k G$ - and $k N$-modules.

Theorem 2.7. [20, Theorem 3.6] Suppose that $S$ is cyclic. Let $\widetilde{S}$ be the unique subgroup of $S$ of order $p$, and let $N=N_{G}(\widetilde{S})$.

(a) $T(G)=\left\{\left[M^{\uparrow G}\right] \mid[M] \in T(N)\right\} \cong T(N)$.

(b) There is an exact sequence

$$
0 \longrightarrow X(N) \longrightarrow T(N) \stackrel{\operatorname{Res}_{S}^{N}}{\longrightarrow} T(S) \longrightarrow 0
$$

where $X(N)$ denotes the group of isomorphism classes of the one dimensional $k N$-modules.

\section{3. $T F(G)$ FOR GENERAL LINEAR GROUPS}

In this section, we begin by introducing some notation and recalling a few facts about the general linear groups and its subgroups. From this information we are able to deduce that the rank of the torsion free part of the group of endotrivial modules 
is always one or zero. The zero case only occurs when the Sylow $p$-subgroup is cyclic. The basic premise of the proof is that every elementary abelian $p$-subgroup of $\operatorname{GL}(n, q)$ is conjugate to a subgroup of a specific standard subgroup. This has been observed before in other contexts. We sketch a proof for the sake of completeness. General reference material can be found in the standard textbooks [2, 18, 23].

We set some notation that is used throughout the paper.

Notation 3.1. Let $n$ is a positive integer, and $q$ a power of a prime such that $\operatorname{gcd}(p, q)=1$ where $p$ is the characteristic of the field $k$. Let $e$ denote the least integer such that $p$ divides $q^{e}-1$. Thus, $e$ is the smallest integer such that $p$ divides the order of $\operatorname{GL}(e, q)$. Let $r, f$ be integers such that $n=r e+f$ for $0 \leq f<e$.

A Sylow $p$-subgroup $S$ of $G$ is a direct product of iterated wreath products. From [18, Section 4.10] a conjugate of $S$ is contained in the normalizer of an appropriate torus of $G$. In particular, for $G=\operatorname{GL}(n, q)$, a Sylow subgroup of $G$ can be realized as the Sylow $p$-subgroup of a semi direct product $\left(C_{p^{t}}\right)^{\times r} \rtimes \mathcal{S}_{r}$, where $\mathcal{S}_{r}$ is the symmetric group on $r$-letters and $p^{t}$ is highest power of $p$ dividing $q^{e}-1$. For $G=\mathrm{SL}(n, q)$, a Sylow $p$-subgroup is the intersection of $G$ with a Sylow $p$-subgroup of $\operatorname{GL}(n, q)$. The exact structure is not important for this paper, except that it is helpful to know the following consequence of these observations.

Lemma 3.2. A Sylow p-subgroup of $\operatorname{GL}(n, q)$ or of $\operatorname{SL}(n, q)$ is abelian if and only if $n<p e$.

We also need the following fact about the elementary abelian $p$-subgroups of $G$. For notation, let $E_{p}$ be an elementary abelian $p$-subgroup of $G=\operatorname{SL}(n, q)$ or $G=\mathrm{GL}(n, q)$ constructed as follows. In the case that $p$ divides $q-1$ (so $e=1$ ), we let $E_{p}$ be the intersection of $G$ with the subgroup of all diagonal matrices whose entries are $p^{t h}$ roots of unity in $\mathbb{F}_{q}$. Otherwise, we let $L=L_{1} \times L_{2} \times \cdots \times L_{r} \times L_{r+1}$ be the Levi subgroup of $G$ consisting of $e \times e$ diagonal blocks, except for the last one which is an $f \times f$ block if $f>0$ and is empty otherwise. Let $E_{p}$ be a maximal elementary abelian $p$-subgroup of $L$. It is the subgroup of elements of order dividing $p$ in a Sylow $p$-subgroup of $L$. It has order $p^{r}$ and is generated by elements that are the identity in all but one of the blocks of $L$. Note that for each $1 \leq i \leq r$ any two subgroups of order $p$ in $L_{i}$ are conjugate since the Sylow $p$-subgroups of $L_{i}$ are cyclic. More extensive details are given in Section 6.

Lemma 3.3. Suppose that $E \subseteq \operatorname{SL}(n, q)$ is an elementary abelian p-subgroup. Then $E$ is conjugate to a subgroup of $E_{p}$.

Proof. Let $\mathbb{V}$ denote the natural module for $G$. The restriction $\mathbb{V}_{E}$ of $\mathbb{V}$ to a $\mathbb{F}_{q} E$ module is completely reducible by Maschke's Theorem. In the case that $p$ divides $q-1$ all irreducible modules have dimension one. The change of basis matrix for the direct sum decomposition conjugates $E$ to a subgroup of $E_{p}$. In the case that $e>1$, the absolutely irreducible characters of $E$ are maps into $\mathbb{F}_{q^{e}}^{\times}$and there is a copy of $\mathbb{F}_{q^{e}}^{\times}$in $\mathrm{SL}(e, q)$. Hence, all of the simple $\mathbb{F}_{q} E$-modules have dimension $e$ or 1. The only one of dimension one is the trivial module. Again, the change of basis matrix that creates the direct sum decomposition conjugates $E$ into an elementary abelian $p$-subgroup of $L$. This can then be conjugated into $E_{p}$. 
This information settles the issue of the torsion free part of the group of endotrivial modules. Note that this theorem does not depend upon the Sylow $p$-subgroup being abelian.

Theorem 3.4. Let $G$ be a group such that $\operatorname{SL}(n, q) \subseteq G \subseteq \operatorname{GL}(n, q)$. Suppose that the Sylow p-subgroup of $G$ has rank at least 2 . Then $T F(G) \cong \mathbb{Z}$ is generated by the class of $\Omega(k)$.

Proof. The proof is a direct consequence of Theorem 2.1 using Lemma 3.3.

We end this section with a few words about Young modules. The setting for general linear groups is described in [16]. Let $G=\operatorname{GL}(n, q)$. A Young module is an indecomposable direct summand of an induced module $k_{L}^{\uparrow G}$ where $L$ is a Levi subgroup of $G$. The Young module corresponding to $L$ is indexed by the same partition of $n$ that determines $L$. That is, if $L=\mathrm{GL}\left(n_{1}, q\right) \times \cdots \times \mathrm{GL}\left(n_{t}, q\right)$, for $n=n_{1}+\cdots+n_{t}$ with $n_{1} \geq \cdots \geq n_{t}$, then for $\lambda=\left[n_{1}, \ldots, n_{t}\right]$, the Young module $Y_{\lambda}$ is a distinguished direct summand of the induced module $k_{L}^{\uparrow G}$. In particular $Y_{[n]}=k$ for the trivial partition $[n]$ of $n$.

The key facts needed about Young modules are summarized in the following theorem.

Theorem 3.5. [16] Suppose that $G=\operatorname{GL}(n, q)$ and $L$ is a Levi subgroup of $G$ corresponding to a partition $\lambda$ of $n$. Then,

$$
k_{L}^{\uparrow G} \cong Y_{\lambda} \oplus \bigoplus_{\mu>\lambda}\left(Y_{\mu}\right)^{\times a_{\mu}},
$$

for some multiplicities $a_{\mu}$. That is, $k_{L}^{\uparrow G}$ is a direct sum of Young modules with exactly one of them being $Y_{\lambda}$. The other direct summands are Young modules that are indexed by partitions which are greater than $\lambda$ in the dominance ordering.

\section{Abelian Sylow $p$-Subgroups When $p$ Divides $q-1$}

In this section we assume that $G=\operatorname{SL}(n, q)$ and that the field $k$ has characteristic $p$ dividing $q-1$. We assume further that the Sylow $p$-subgroup of $G$ is abelian and not cyclic. This requires that $2<n<p$. Our goal is to show that every indecomposable $k G$-module with trivial Sylow restriction has dimension one.

Let $T$ be the torus of diagonal matrices with determinant one, and let $N$ be its normalizer in $G$. It is well known that the Weyl group $N / T$ is isomorphic to the symmetric group $\mathcal{S}_{n}$. By [18, Theorem 4.10.2], our hypotheses also says that $T$ contains a Sylow $p$-subgroup $S$ of $G$. The first result is crucial to our arguments.

Proposition 4.1. Suppose that $M$ is a $k G$-module with trivial Sylow restriction. Then the restriction of $M$ to $T$ has the form, $M_{\downarrow T} \cong k \oplus$ (proj).

Proof. Note that the Sylow subgroup $S$ of $T$ is normal in $N$. Consequently, $M_{\downarrow N} \cong$ $X \oplus R$ where $X$ has dimension one and $R$ is a projective $k N$-module. We observe that $M_{\downarrow T} \cong X_{\downarrow T} \oplus R_{\downarrow T}$, and $R_{\downarrow T}$ is a projective $k T$-module. So the proof is complete if we show that $X_{\downarrow T}$ is a trivial $k T$-module. 
The representation $N \longrightarrow \mathrm{GL}(X)$ that affords $X$ has the commutator subgroup $[N, N]$ of $N$ in its kernel, since $\operatorname{GL}(X)$ is commutative. Choose a generator $\zeta$ for the multiplicative group $\mathbb{F}_{q}^{\times}$of nonzero elements of $\mathbb{F}_{q}$. The group $T$ is generated by diagonal matrices $D_{1}, \ldots, D_{n-1}$ where $D_{i}$ has diagonal entries $1, \ldots, 1, \zeta, \zeta^{-1}, 1, \ldots, 1$, the entry $\zeta$ being in the $i^{t h}$ position. Let $\sigma_{i}$ be the block matrix

$$
\sigma_{i}=\left(\begin{array}{ccc}
I_{i-1} & 0 & 0 \\
0 & U & 0 \\
0 & 0 & I_{n-i-1}
\end{array}\right)
$$

where $I_{j}$ is the $j \times j$ identity matrix and

$$
U=\left(\begin{array}{cc}
0 & -1 \\
1 & 0
\end{array}\right) .
$$

If $i<n-1$, let $W=D_{i+1}$, and if $i=n-1$, let $W=D_{i-1}$. We then get the equality $\sigma_{i} W \sigma_{i}^{-1} W^{-1}=D_{i}$. Therefore $T \subseteq[N, N]$, and $T$ acts trivially on $X$. This completes the proof.

For later use we record the following lemma which is proved above.

Lemma 4.2. Suppose that $G=\operatorname{SL}(n, q)$ where $p$ divides $q-1$ and $3 \leq n<p$. Then for $T$ and $N$ as above, we have that $T \subseteq[N, N]$.

Next, let $L=(\operatorname{GL}(n-1, q) \times \mathrm{GL}(1, q)) \cap G$ be the Levi subgroup of all matrices of determinant one that can be put into block form of an upper left $(n-1) \times(n-1)$ block and a lower right $1 \times 1$ block.

Proposition 4.3. Assume that $p$ divides $q-1$. Let $M$ be a $k G$-module with trivial Sylow restriction. Then the restriction of $M$ to $L$ has the form, $M_{\downarrow L} \cong k_{L} \oplus$ (proj).

Proof. Suppose that $\zeta \in \mathbb{F}_{q}$ is a primitive $p^{\text {th }}$ root of unity. Let $z \in G$ be the diagonal matrix with diagonal entries $\zeta, \zeta, \ldots, \zeta, \zeta^{1-n}$. Note that $z \notin Z(G)$ because our assumption $2<n<p$ implies that $1-n \not \equiv 1(\bmod p)$. However the important point is that $z$ generates a subgroup of order $p$ that is central in $L$. In particular, $L$ has a nontrivial normal $p$-subgroup. Hence, $M_{\downarrow L} \cong X \oplus$ (proj) where $X$ has dimension one by Proposition 2.3. By Proposition 4.1, it suffices to show that the restriction of $X$ to $T$ is the trivial module if and only if $X$ is the trivial $k L$-module.

We observe that the homomorphism $\psi: \operatorname{GL}(n-1, q) \longrightarrow \operatorname{SL}(n, q)$, given by sending an invertible matrix $A$ to the matrix

$$
A \mapsto\left(\begin{array}{cc}
A & 0 \\
0 & \operatorname{Det}(A)^{-1}
\end{array}\right),
$$

induces an isomorphism $\mathrm{GL}(n-1, q) \cong L$. The one dimensional representations of $\mathrm{GL}(n-1, q)$ over $k$ are well known and are the maps

$$
\varphi_{i}: \operatorname{GL}(n-1, q) \longrightarrow k^{\times} \quad \text { given by } A \mapsto \operatorname{Det}(A)^{i}
$$

for $i=0,1, \ldots, q-2$. It is easy to see that the only $\varphi_{i}$ which has $T$ in its kernel is $\varphi_{0}$. This finishes the proof. 
Before proceeding to our main result, we record a known fact involving the numbers of cosets for an arbitrary group $H$ that will be needed later. We give a sketch of the proof for the sake of completeness.

Lemma 4.4. Suppose that $U$ and $V$ are subgroups of a finite group $H$ such that $U \subseteq V$. Then the number of $U-V$ double cosets $U x V$ in $H$ that are left cosets of $V$ is the same as the number of double cosets $U x V$ in $H$ such that $U \subseteq x V x^{-1}$. This number is at least as large as $|V| /\left|N_{V}(U)\right|$.

Another way to cast Lemma 4.4 is to say that the number of left cosets $x V$ of $H / V$ that are stabilized by the action of $U$ (on the left) is equal to the index of $N_{V}(U)$ in $V$.

Proof. Note that for $x$ in $H$, we have that $U x V$ is a left coset of $V$ in $H$ if and only if $U x V=x V$. This is equivalent to saying that $x^{-1} U x \subseteq V$ or that $U \subseteq x V x^{-1}$. Now note that if $x \in N_{H}(U)$, then $U x V=x V$. Hence, the number of such double cosets is at least equal to

$$
\frac{\left|N_{H}(U) \cdot V\right|}{|V|}=\frac{|V|}{\left|N_{H}(U) \cap V\right|}=\frac{|V|}{\left|N_{V}(U)\right|}
$$

Proposition 4.3 shows that if $p$ does not divide $n$ and if $M$ is an indecomposable $k G$-module with trivial Sylow restriction then the restriction of $M$ to $L$ is the direct sum of a trivial module and a projective module. Because $L$ contains a Sylow $p$ subgroup of $G$, it follows from standard arguments on relative projectivity that $M$ is a direct summand of $k_{L}^{\uparrow G}$, the induction of the trivial $k L$-module to $G$. Now by the theory of Young modules for the general linear group (see Theorem 3.5), we have that

$$
k_{\widehat{L}}^{\uparrow \widehat{G}} \cong Y_{[n-1,1]} \oplus Y_{[n]},
$$

where $\widehat{G}=\operatorname{GL}(n, q)$ and $\widehat{L}=\operatorname{GL}(n-1, q) \times \operatorname{GL}(1, q)$ is the Levi subgroup in $\widehat{G}$. Indeed Theorem 3.5 asserts that $k_{\widehat{L}}^{\uparrow \widehat{G}} \cong Y_{[n-1,1]} \oplus\left(Y_{[n]}\right)^{\times m}$ for some $m \geq 1$, because $[n]$ is the only partition of $n$ greater than $[n-1,1]$. Now $Y_{[n]}=k$ and so we get that $m=1$ by Frobenius reciprocity. Hence once we show that the restriction to $G$ of the indecomposable module $Y_{[n-1,1]}$ has no endotrivial direct summands we will be able to deduce the following result.

Theorem 4.5. Suppose that $G=\operatorname{SL}(n, q)$ with $2<n<p$ and that $p$ divides $q-1$. Then $T T(G)=\{0\}$.

Proof. Recall from Section 3 that the assumption $2<n<p$ says that a Sylow $p$-subgroup $S$ of $G$ is abelian and not cyclic. More precisely, if $p^{t}$ is the highest power of $p$ dividing $q-1$, then $S \cong\left(C_{p^{t}}\right)^{n-1}$ is homocyclic. For convenience, let $\widehat{G}=\operatorname{GL}(n, q)$ and $\widehat{L} \cong \operatorname{GL}(n-1, q) \times \operatorname{GL}(1, q)$ for the usual Levi subgroup and set $L=\widehat{L} \cap G$. Note that $L \cong \operatorname{GL}(n-1, q)$ has index $q-1$ in $\widehat{L}$. We choose $S \leq T$, so that $N_{G}(S) \leq N$. By Proposition 4.3, the group $T T(G)$ is generated by the classes of the indecomposable endotrivial modules that are direct summands of the induced module $k_{L}^{\uparrow G}$. 
We start by noticing that $k_{L}^{\uparrow G} \cong\left(k_{\widehat{L}}^{\uparrow \widehat{G}}\right)_{\downarrow G}$. In order to see this, apply the Mackey formula to

$$
\left(k_{\widehat{L}}^{\uparrow \widehat{G}}\right)_{\downarrow G} \cong \sum_{g \in[G \backslash \widehat{G} / \widehat{L}]}\left(k_{g \widehat{L} \cap G}\right)^{\uparrow G}=k_{L}^{\uparrow G} .
$$

The last equality is a consequence of the facts that $\widehat{L} G=\widehat{G}$ and there is only one $\widehat{L}-G$ double coset in $\widehat{G}$, represented by $g=1$.

From Equation (2)

$$
k_{\widehat{L}}^{\uparrow \widehat{G}} \cong Y_{[n-1,1]} \oplus Y_{[n]}
$$

so that $k_{L}^{\uparrow G} \cong\left(Y_{[n-1,1]}\right)_{\downarrow G} \oplus k$. We are left with the task of proving that $\left(Y_{[n-1,1]}\right)_{\downarrow G}$ has no endotrivial direct summands.

Now let $U=\langle\sigma\rangle$ with $\sigma \in G$ the diagonal matrix with entries $\zeta^{1-n}, \zeta, \ldots, \zeta$ where $\zeta$ is a primitive $(q-1)$-st root of unity. Because $2<n<p$, we have that $\zeta \neq \zeta^{1-n}$ and the normalizer $N_{L}(U)$ of $U$ in $L$ is the intersection of the Levi subgroup $\operatorname{GL}(1, q) \times \operatorname{GL}(n-1, q)$ of $\widehat{G}$ with $L$. Hence, the intersection is isomorphic to $\operatorname{GL}(1, q) \times \operatorname{GL}(n-2, q)$, and

$$
N_{G}(U)=(\mathrm{GL}(1, q) \times \mathrm{GL}(n-1, q)) \cap G \cong \mathrm{GL}(n-1, q) .
$$

We claim that $\left(Y_{[n-1,1]}\right)_{\downarrow U}$ is not an endotrivial $k U$-module. The Mackey formula yields

By Lemma 4.4

$$
\left(k_{L}^{\uparrow G}\right)_{\downarrow U} \cong \sum_{g \in[U \backslash G / L]}\left(k_{g L \cap U}\right)^{\uparrow U}
$$

$$
|[U \backslash G / L]| \geq\left|L: N_{L}(U)\right|>2
$$

and so there are at least three trivial direct summands in $\left(k_{L}^{\uparrow G}\right)_{\downarrow U} \cong\left(Y_{[n-1,1]}\right)_{\downarrow U} \oplus k_{\downarrow U}$. This is because we can choose double coset representatives for $[U \backslash G / L]$ in $N_{G}(U)$, which implies that ${ }^{g} L \cap U=U$ for each $g \in[U \backslash G / L]$. So the multiplicity of $k_{U}$ as a direct summand of $\left(Y_{[n-1,1]}\right)_{\downarrow U}$ is at least two, which proves that $\left(Y_{[n-1, n]}\right)_{\downarrow U}$ is not endotrivial.

We claim that this is enough to prove the theorem. Suppose that $\left(Y_{[n-1, n]}\right)_{\downarrow G}$ has an endotrivial direct summand. To show the claim note that $S$ is characteristic in $T$ and hence normal in $N$. So any endotrivial $k N$-module having trivial Sylow restriction has dimension one.

Since $N \cong T \rtimes \mathcal{S}_{n}$ and $T \subseteq[N, N]$, there are exactly two distinct one dimensional $k N$-modules, namely $k$ and the sign module. Then $k_{T}^{\uparrow N}$ has exactly two one-dimensional direct summands. As a consequence, $k_{T}^{\uparrow G}=\left(k_{T}^{\uparrow N}\right)^{\uparrow G}$ can have at most two endotrivial direct summands (the Green correspondents of the one dimensional modules). Now $k_{L}^{\uparrow G}$ is a direct summand of $k_{T}^{\uparrow G}$, so it can have at most two endotrivial directs summand, one of which is $k$.

By Clifford theory, $\left(Y_{[n-1,1]}\right)_{\downarrow G}$ is either indecomposable or the direct sum of conjugate modules. If the latter holds, then one of the summands is endotrivial and the conjugates are also. We know that $\left(Y_{[n-1,1]}\right)_{\downarrow G}$ can have only one endotrivial indecomposable direct summand and $\left(Y_{[n-1,1]}\right)_{\downarrow G}$ must be indecomposable and endotrivial. However, this is not possible as the restriction to $U$ is not endotrivial. 


\section{THE KERNEL OF RESTRICTION}

In this section we present a method introduced by Balmer [3] (see also [4]) for computing the kernel of the restriction map $T(G) \rightarrow T(H)$ in the case that $H \subseteq$ $G$ is a subgroup that contains a Sylow $p$-subgroup of $G$. We also develop some consequences of these basic ideas that will be used in later sections. The main definition is the following.

Definition 5.1. Suppose that $H$ is a subgroup of $G$ that contains $S$, a Sylow psubgroup of $G$. A function $u: G \rightarrow k^{\times}$is a weak $H$-homomorphism if it satisfies the three conditions:

(a) if $h \in H$, then $u(h)=1$,

(b) for $g \in G$, if $\left|H \cap{ }^{g} H\right|$ has order prime to $p$, then $u(g)=1$, and

(c) if $a, b \in G$ and $p$ divides $\left|H \cap{ }^{a} H \cap{ }^{a b} H\right|$ then $u(a b)=u(a) u(b)$.

Let $A(G, H)$ be the set of all weak $H$-homomorphisms. It is easy to show that $u\left(g^{-1}\right)=(u(g))^{-1}$ for every $u \in A(G, H)$ and all $g \in G$. Furthermore, one can verify that the set $A(G, H)$ is a group under composition of functions. If $G$ has a nontrivial normal $p$-subgroup, then every weak $H$-homomorphism is a homomorphism whose kernel contains $H$. Balmer proved an amazing theorem which relates $A(G, H)$ and the kernel of the restriction map from $T(G)$ to $T(H)$.

Theorem 5.2. [3] Suppose that $H$ is a subgroup of $G$ of index prime to $p$. Then $A(G, H)$ is isomorphic to the kernel of the restriction map $T(G) \rightarrow T(H)$.

We use that theorem to establish two sets of criteria for the vanishing of the kernel of restriction on endotrivial modules. First we need the following basic lemma. Part (a) is proven in [3]. We include a proof for the convenience of the reader.

Lemma 5.3. Let $H$ be a subgroup of $G$ having index prime to $p$. Suppose that $u: G \rightarrow k^{\times}$is a weak H-homomorphism. Then

(a) $u$ is constant on $\mathrm{H}-\mathrm{H}$ double cosets (meaning that if $\mathrm{HaH}=\mathrm{HbH}$ then $u(a)=u(b))$,

(b) if $a, b \in N_{G}(Q)$ for some nontrivial p-subgroup $Q \subseteq H$, then $u(a b)=$ $u(a) u(b)$, and

(c) if $g$ is in the commutator subgroup $[N, N]$ of $N=N_{G}(Q)$, for some nontrivial p-subgroup $Q \subseteq H$, then $u(g)=1$.

Proof. Note that part (b) follows directly from the definition of a weak $H$-homomorphism, and part (c) is a trivial consequence of (b). So only part (a) needs some proof. For this assume that $a, b \in G$ and that $H a H=H b H$, so that $b=h_{1} a h_{2}$ for some $h_{1}, h_{2} \in H$. There are two cases to consider.

First, if $\left|H \cap{ }^{a} H\right|$ is prime to $p$ then so also is $\left|H \cap{ }^{h_{1} a h_{2}} H\right|=\left|\left(H \cap{ }^{a} H\right)^{h_{1}^{-1}}\right|$. So the lemma holds in this case.

Second, suppose that $\left|H \cap{ }^{a} H\right|$ is divisible by $p$. Then clearly, $\left|H \cap{ }^{h_{2}} H \cap{ }^{a h_{2}} H\right|$ has order divisible by $p$. So $u\left(a h_{2}\right)=u(a)$. Also, there exists a nontrivial $p$-subgroup $Q$ in $H \cap{ }^{a} H$. Thus ${ }^{h_{1}} Q$ is in $H \cap{ }^{h_{1}} H \cap{ }^{h_{1} a} H$. So $u\left(h_{1} a\right)=u(a)$, as desired. 
Our first application is almost an immediate consequence of the above lemma, in particular parts (a) and (c).

Proposition 5.4. Let $H$ be a subgroup of $G$ having index relatively prime to $p$. Suppose that every $H-H$ double coset $H x H$ in $G$ with $x \notin H$ has the property that either

(a) $p$ does not divide $\left|H \cap{ }^{x} H\right|$, or

(b) $H x H=H a H$ for some a in $\left[N_{G}(Q), N_{G}(Q)\right]$, the commutator subgroup of $N_{G}(Q)$, for some nontrivial p-subgroup $Q$ of $H$.

Then $A(G, H)=\{1\}$.

The next application applies only to the case that $H=S$ is a Sylow $p$-subgroup of $G$.

Proposition 5.5. Let $S$ be a Sylow p-subgroup of $G$. Suppose that the following two conditions are satisfied.

(a) For any element $u$ in $A(G, S)$, we have that $u(x)=1$ whenever $x \in N_{G}(Q)$ for some nontrivial subgroup $Q$ of $S$.

(b) If $Q_{1}$ and $Q_{2}$ are subgroups of $S$ that are conjugate in $G$, then for some nontrivial subgroup $Q$ of $S$, there exists an element $g \in N_{G}(Q)$ such that ${ }^{g} Q_{1}=Q_{2}$.

Then $A(G, S)=\{1\}$.

Proof. Let $x$ be any element of $G$ such that $S \cap{ }^{x} S=Q \neq\{1\}$. Our purpose is to show for any $u \in A(G, S)$, that $u(x)=1$. This would prove the proposition. There are exactly two things that can happen in this situation. The first is that $x \in N_{G}(Q)$, in which case $u(x)=1$ by condition (a) of the hypothesis. Hence, we are left to assume the second possibility that ${ }^{x} Q \neq Q$. Let $R={ }^{x^{-1}} Q$. Then ${ }^{x} R=Q$. By condition (b) of the hypothesis, there exists an element $g \in N_{G}(\widehat{Q})$ for some nontrivial subgroup $\widehat{Q}$ of $S$ such that ${ }^{g} Q=R$. Note, that by condition (a), $u(g)=1$. Then we have that $R={ }^{g} Q={ }^{x^{-1}} Q$, so that $x g \in N_{G}(Q)$. Thus, $u(x g)=1$ by condition (a). Now note that $S \cap{ }^{x} S \cap{ }^{x g} S \supseteq Q$ which is not trivial. So from the definition we have that $u(x g)=u(x) u(g)$ and $u(x)=1$ as desired.

In special cases, we can relax the conditions somewhat as in the following.

Corollary 5.6. Suppose that the Sylow p-subgroup $S$ of $G$ is abelian. Let $E$ be the subgroup of $S$ of all elements with order $p$ (or 1 ). Assume also that the following condition is satisfied.

- For any element $u$ in $A(G, S)$, we have that $u(x)=1$ whenever $x \in N_{G}(Q)$ for some nontrivial subgroup $Q \subseteq E$.

Then $A(G, S)=\{1\}$.

Proof. Observe that if $Q$ is any nontrivial subgroup of $S$, then the $\operatorname{subgroup} Q \cap E$, which contains all elements of order $p$ in $Q$, has the property that $N_{G}(Q) \subseteq N_{G}(Q \cap$ $E)$. Hence, the hypothesis implies condition (a) of Proposition 5.5. Condition (b) is a consequence of the fusion theorem of Burnside (see [17, Chapter 7, Theorem 
1.1]), which says that, because $S$ is abelian, $N_{G}(S)$ controls the fusion of subgroups of $S$.

We end this section with a proof of an inductive step in a scheme to use the above results effectively. For notation, let $\Gamma_{G}$ denote the set of nontrivial subgroups of $G$, and let $\Gamma_{H, p}$ denote the set of nontrivial $p$-subgroups of $H$.

Proposition 5.7. Let $H \subseteq G$ be a subgroup of index relatively prime to $p$. Suppose that $\rho: \Gamma_{H, p} \rightarrow \Gamma_{G}$ is a function with the properties:

(a) for every $Q \in \Gamma_{H, p}, \rho(Q) \subseteq N_{G}(Q)$, and

(b) if $u \in A(G, H)$ and $Q \in \Gamma_{H, p}$, then $u(g)=1$ for all $g \in \rho(Q)$.

Let $\widehat{\rho}: \Gamma_{H, p} \rightarrow \Gamma_{G}$ be defined by the rule

$$
\widehat{\rho}(Q)=\left\langle N_{G}(Q) \cap \rho\left(Q^{\prime}\right) \mid Q^{\prime} \in \Gamma_{H, p}\right\rangle,
$$

the subgroup generated by $\rho(Q)$ and all of the intersections $N_{G}(Q) \cap \rho\left(Q^{\prime}\right)$ for $Q^{\prime}$ in $\Gamma_{H, p}$. Then $\widehat{\rho}$ also satisfies the above properties. Namely,

(i) for every $Q \in \Gamma_{H, p}$, we have that $\widehat{\rho}(Q) \subseteq N_{G}(Q)$, and

(ii) if $u \in A(G, H)$ and $Q \in \Gamma_{H, p}$, then $u(g)=1$ for all $g \in \widehat{\rho}(Q)$.

Proof. That $\widehat{\rho}(Q) \subseteq N_{G}(Q)$ for all $Q \in \Gamma_{H, p}$ is obvious from the construction. To establish the second property, assume that $g \in \widehat{\rho}(Q)$ for some $Q$. Then $g=g_{1} g_{2} \ldots g_{n}$ for some $n$ and for $g_{i} \in N_{G}(Q) \cap \rho\left(Q_{i}\right)$ for some $Q_{i} \in \Gamma_{H, p}$. Thus, $u\left(g_{i}\right)=1$ for all $i=1, \ldots, n$. But because all of these elements are in $N_{G}(Q)$, we have that $u(g)=u\left(g_{1}\right) \cdots u\left(g_{n}\right)=1$.

Remark 5.8. The function $\rho: \Gamma_{H, p} \rightarrow \Gamma_{G}$ given by $\rho(Q)=\left[N_{G}(Q), N_{G}(Q)\right]$ satisfies the hypothesis of the proposition by Lemma 5.3(c).

\section{Some information about $\operatorname{GL}(e, q)$}

The objective of this section is to set some notation for the remainder of the paper and to establish some elementary facts about the group $G=\operatorname{GL}(e, q)$. We include sketches of some proofs for the sake of completeness.

Throughout the section, let $\mathbb{F}=\mathbb{F}_{q}$ and $\mathbb{K}=\mathbb{F}_{q^{e}}$, and assume Notation 3.1. In addition, assume throughout the section that $e>1$. Notice that this assumption requires that $p>2$. Thus, $p$ divides $q^{e-1}+\cdots+q+1$.

Let $\mathbb{V}$ denote the natural module for $G$. We can identify $\mathbb{V}$ with $\mathbb{K}$, and we have an $\mathbb{F}$-linear action of $\mathbb{K}^{\times}$on itself. Consequently, we have a $\mathbb{F}$-linear embedding of $\mathbb{K}^{\times}$into $G$. There is an action of the Galois group of $\mathbb{K}$ over $\mathbb{F}$ by $\mathbb{F}$-linear transformation. Consequently, the Galois group can also be embedded in $G$.

Notation 6.1. Let $w$ be a generator for $\mathbb{K}^{\times}$, regarded as a subgroup of $G$. Let $u \in\langle w\rangle$ be an element of order $p$. Let $g \in G$ denote a generator for the Galois group of $\mathbb{K}$ over $\mathbb{F}$, so that $g w g^{-1}=w^{q}$. Let $v$ be an element of $C_{G}(S)$ of determinant -1 . (The existence of such an element $v$ is established in Lemma 6.2(c).)

We assume that $u$ is the companion matrix of its minimal polynomial over $\mathbb{F}_{q}$. The Sylow subgroup $S$ of $G$ is cyclic since the power of $p$ dividing $|G|$ is the same as that dividing $\left|\mathbb{K}^{\times}\right|$which is cyclic. Hence, we can consider $S$ to be a subgroup of 
$\langle w\rangle=\mathbb{K}^{\times} \subseteq G$, and $u \in S$. Note, however, that $S$ need not be generated by $u$. For an example, let $G=\mathrm{GL}(5,3)$ with $p=11$. In this case, $11^{2}$ divides $3^{5}-1$, but 11 does not divide $3^{t}-1$ for $t<5$.

Lemma 6.2. With the above notation, and assuming that $e>1$, we have the following.

(a) The centralizer of $u$ in $G$ is $\langle w\rangle$.

(b) The normalizer of $S$ in $G$ is generated by $w$ and $g$.

(c) The restriction of the determinant function Det : $G \rightarrow \mathbb{F}_{q}^{\times}$to $\mathbb{K}^{\times} \subseteq G$ is surjective. Specifically, $\operatorname{Det}(w)=w^{s}$ where $s=1+q+\cdots+q^{e-1}$ and $w^{s}$ is a generator for the cyclic group $\mathbb{F}_{q}^{\times}$.

(d) The commutator subgroup of $N_{G}(S)$ is generated by $w^{q-1}$. It has index $e(q-1)$ in $N_{G}(S)$ and is equal to the set of all elements of $C_{G}(S)$ that have determinant 1.

(e) The determinant of $u$ is 1 , and the determinant of $g$ is $(-1)^{e-1}$.

(f) Suppose that a is an $e \times e$ matrix over $\mathbb{F}_{q}$ such that au $u^{\ell}=u^{m} a$ for some $\ell, m$ such that $\ell$ is not divisible by $p$. Then either $a$ is invertible or $a=0$.

Proof. We note that the natural module $\mathbb{V}$ is an irreducible module for the group of order $p$ generated by $u$. This is because an element of order $p$ acts on no smaller $\mathbb{F}_{q}$-vector space by the minimality of $e$. So by Schur's Lemma, the commuting ring of the action is a field, and we know that this field contains $\mathbb{K}$. On the other hand the algebra of $e \times e$ matrices over $\mathbb{F}_{q}$ contains no extensions of degree greater than $e$. This proves (a).

For (b), suppose that $x \in G$ normalizes $S$. Then conjugation by $x$ is an automorphism on the subalgebra of $e \times e$ matrices generated by $w$, which is isomorphic to $\mathbb{K}$. This requires that $x$ act on $w$ as some power of $g$, or that $x$ be equal to some power of $g$ times an element of the centralizer of $w$ which is $\langle w\rangle$.

The minimal polynomial of $w$ over $\mathbb{F}_{q}$ is the product $f(X)=\prod_{i=0}^{e-1}\left(X-w^{q^{i}}\right)$. That is, the roots of this polynomial are $w$ and its Galois conjugates. The constant term of $f(X)$, is $(-1)^{e} w^{s}$. The matrix of $w$ over $\mathbb{F}_{q}$ is conjugate to the companion matrix of its minimal polynomial whose determinant is $(-1)^{e}$ times the constant term of the polynomial. Hence, we have that $\operatorname{Det}(w)=w^{s}$, as asserted. The order of $w$ is $\left|\mathbb{K}^{\times}\right|=q^{e}-1=(q-1) s$. Consequently, $w^{s}$ has order $q-1$ and $w^{s}$ is a generator for $\mathbb{F}_{q}^{\times}$. This proves (c).

If $x$ is in $\langle w\rangle=C_{G}(S)$, then $g x g^{-1} x^{-1}=x^{q} x^{-1}=x^{q-1}$. Thus, $\left[N_{G}(S), N_{G}(S)\right]=$ $\left\langle w^{q-1}\right\rangle$ as asserted. The second statement in (d) follows from (c).

The first statement of (e) is a consequence of the fact that $p$ does not divide $q-1$. For the second, we note that there is a basis of $\mathbb{K}$ as an $\mathbb{F}_{q}$-vector space that is permuted by the Galois automorphism $g$. Consequently, viewing the natural module $\mathbb{V}$ as $\mathbb{K}$, with respect to this basis, the element $g$ acts by a permutation matrix, representing an $e$-cycle.

For (f) we observe that $a u^{\ell}=u^{m} a$ implies that $u^{\ell}$ stabilizes the nullspace of $a$. However, we know that the group $\left\langle u^{\ell}\right\rangle=\langle u\rangle$ has no nontrivial $\mathbb{F}_{q}$ representations of degree less than $e$. So either the nullspace of $a$ is zero or it is the entire natural module. 


\section{Endotrivial modules for $\operatorname{SL}(2 e, q)$}

In this section we prove that $T T(G)=\{0\}$ for $G=\operatorname{SL}(2 e, q)$, where $e$ is the least integer such that $p$ divides $q^{e}-1$ and $p>2$. Our strategy is to show that the group of weak $S$-homomorphisms $A(G, S)$ is trivial. This implies the kernel of the restriction map is trivial and the restriction map from $T(G) \rightarrow T(S)$ is a monomorphism where $S$ is a Sylow $p$-subgroup of $G$. In the proof we verify the condition in Corollary 5.6, by an inductive process described in Proposition 5.7. This requires a detailed analysis of the normalizers of the $p$-subgroups in $S$.

For notational convenience, we express elements of $G$ as $2 \times 2$ block matrices where the blocks have size $e \times e$, and keep the notation of the previous section. In particular, the elements $w, u, g$ and $v$ are as in Notation 6.1. Let $H$ denote the group GL $(e, q)$. The Sylow $p$-subgroup $S$ of $G$ has order $p^{2 t}$ where $p^{t}$ is the highest power of $p$ dividing $q^{e}-1$. The group $S$ is the direct product of two cyclic groups of order $p^{t}$, and has a maximal elementary abelian subgroup $E$ of order $p^{2}$. We can assume $E$ is generated by

$$
A=\left[\begin{array}{ll}
u & 0 \\
0 & 1
\end{array}\right] \text { and } B=\left[\begin{array}{ll}
1 & 0 \\
0 & u
\end{array}\right] .
$$

The subgroups of order $p$ in $E$ are naturally divided into three types. We give representatives as

$$
\text { (1.) } Q_{1}=\langle A\rangle, \quad \text { (2.) } Q_{2}=\langle A B\rangle \text {, and (3.) } Q_{3}=\left\langle A B^{m}\right\rangle \text {, }
$$

where in the third case, $m$ is an integer such that $u^{m}$ is not conjugate to $u$ in $\operatorname{GL}(e, q)$. Note that in some situations such as when $G=\operatorname{SL}(4,5)$ and $p=3$, there is no such $m$ and the third case does not exist. Note further that $Q_{1}$ is conjugate to $\widehat{Q}_{1}=\langle B\rangle$ and that $Q_{2}$ is conjugate to $\left\langle A B^{q^{\ell}}\right\rangle$ for any $\ell$. Hence, we have the following.

Lemma 7.1. Every subgroup of order $p$ in $G$ is conjugate to $Q_{1}$ or to $Q_{2}$ or to a subgroup of $E$ of type $Q_{3}$.

There is one particular subgroup that plays an important role in this development. Namely,

$$
U=\left\{\left[\begin{array}{ll}
a & 0 \\
0 & b
\end{array}\right] \mid a, b \in C_{H}(u)=\langle w\rangle, \operatorname{Det}(a b)=1\right\} .
$$

We note that $U$ is in the centralizer of every subgroup of $S$.

First we consider the centralizers of these subgroups. Recall that $H=\mathrm{GL}(e, q)$.

Proposition 7.2. The centralizers of the subgroups of order $p$ are given as follows.

$$
\begin{aligned}
C_{G}\left(Q_{1}\right) & =\left\{\left[\begin{array}{ll}
a & 0 \\
0 & b
\end{array}\right] \mid a \in C_{H}(u), b \in H \quad \text { and } \quad \operatorname{Det}(a) \operatorname{Det}(b)=1\right\} . \\
C_{G}\left(Q_{2}\right) & =\left\{X=\left[\begin{array}{ll}
a & b \\
c & d
\end{array}\right] \mid a, b, c, d \in C_{H}(u) \cup\{0\} \quad \text { and } \operatorname{Det}(X)=1\right\} . \\
C_{G}\left(Q_{3}\right) & =U .
\end{aligned}
$$

In the second case, $C_{G}\left(Q_{2}\right)$ is a subgroup of $\mathrm{GL}(2, \mathbb{K})$ that contains $\mathrm{SL}(2, \mathbb{K})$ as a subgroup of index $q-1$. 
Proof. The proofs are straightforward exercises. One note is that in the statement for $C_{G}\left(Q_{2}\right)$ by Lemma $6.2(\mathrm{f})$, each of $a, b, c, d$ is either invertible or is zero. The embedding of $\mathrm{GL}(2, \mathbb{K})$, is defined by the embedding of $\mathbb{K}$ in $H$ as observed in the previous section. The subgroup $\mathrm{SL}(2, \mathbb{K})$ is the commutator subgroup of $\mathrm{GL}(2, \mathbb{K})$ and any commutator (as a $2 e \times 2 e$ matrix) must have determinant one. Consequently, $\mathrm{SL}(2, \mathbb{K})$ is contained in $C_{G}\left(Q_{2}\right)$. The fact about the index is a consequence of Lemma 6.2(c).

Corollary 7.3. The centralizer of $S$ and of $E$ is $C_{G}(S)=C_{G}(E)=U$.

Proof. The centralizer of $E$ is the intersection of the centralizers of $Q_{1}$ and of $\widehat{Q}_{1}=$ $\langle B\rangle$.

Now recall from the last section that $v \in C_{H}(u)=\langle w\rangle$ is an element with $\operatorname{Det}(v)=-1$, and $g \in N_{H}(\langle u\rangle)$ is an element that acts on $\mathbb{K}^{\times}=\langle w\rangle$, as the Galois automorphism, $g u g^{-1}=u^{q}$.

Proposition 7.4. The normalizers of the subgroups of order $p$ in $S$ are given as follows.

(a) $N_{G}\left(Q_{1}\right)$ is generated by $C_{G}\left(Q_{1}\right)$ and by the element $\left[\begin{array}{cc}g & 0 \\ 0 & v^{e-1}\end{array}\right]$.

(b) $N_{G}\left(Q_{2}\right)$ is generated by $C_{G}\left(Q_{2}\right)$ and by the element $\left[\begin{array}{ll}g & 0 \\ 0 & g\end{array}\right]$.

(c) $N_{G}\left(Q_{3}\right)$ is generated by $C_{G}\left(Q_{3}\right)$ and by the element $\left[\begin{array}{ll}g & 0 \\ 0 & g\end{array}\right]$, and possibly also an element of the form $\left[\begin{array}{cc}0 & g^{i} \\ g^{j} v^{e(i+j+1)} & 0\end{array}\right]$.

Also, $N_{G}(S)$ is generated by $C_{G}(S)=U$ and the elements

$$
\left[\begin{array}{cc}
g & 0 \\
0 & v^{e-1}
\end{array}\right] \quad \text { and } \quad\left[\begin{array}{cc}
0 & 1 \\
-1 & 0
\end{array}\right]
$$

Proof. The normalizer $N_{G}\left(Q_{1}\right)$ also normalizes the commutator subgroup of the centralizer $C_{G}\left(Q_{1}\right)$ which has the form

$$
\left[C_{G}\left(Q_{1}\right), C_{G}\left(Q_{1}\right)\right]=\left\{\left[\begin{array}{ll}
1 & 0 \\
0 & b
\end{array}\right] \mid b \in \operatorname{SL}(e, q)\right\} .
$$

The normalizer of $\left[C_{G}\left(Q_{1}\right), C_{G}\left(Q_{1}\right)\right]$ is the Levi subgroup of two $e \times e$ blocks. Then the normalizer of $Q_{1}$ is the subgroup consisting of all elements of the form

$$
\left[\begin{array}{ll}
a & 0 \\
0 & b
\end{array}\right]
$$

such that $a \in N_{H}(\langle u\rangle), b \in H$ and $\operatorname{Det}(a) \operatorname{Det}(b)=1$ (recall that $H=\operatorname{GL}(e, q)$ ). This proves (a).

Any element in $N_{G}\left(Q_{2}\right)$ has the form

$$
\left[\begin{array}{ll}
a & b \\
c & d
\end{array}\right]
$$


and satisfies

$$
\left[\begin{array}{ll}
a & b \\
c & d
\end{array}\right]\left[\begin{array}{ll}
u & 0 \\
0 & u
\end{array}\right]=\left[\begin{array}{cc}
u^{\tau} & 0 \\
0 & u^{\tau}
\end{array}\right]\left[\begin{array}{ll}
a & b \\
c & d
\end{array}\right]
$$

for some value of $\tau$. Thus, $x u=u^{\tau} x$ for $x=a, b, c$ or $d$, and there exists some $\nu$ such that

$$
\left[\begin{array}{ll}
a & b \\
c & d
\end{array}\right]=\left[\begin{array}{cc}
g^{\nu} & 0 \\
0 & g^{\nu}
\end{array}\right] X
$$

where $X \in C_{G}\left(Q_{2}\right)$. So, we have shown (b).

Similarly, in case (c), for some $\tau$, we have

$$
\left[\begin{array}{ll}
a & b \\
c & d
\end{array}\right]\left[\begin{array}{cc}
u & 0 \\
0 & u^{r}
\end{array}\right]=\left[\begin{array}{cc}
u^{\tau} & 0 \\
0 & u^{r \tau}
\end{array}\right]\left[\begin{array}{ll}
a & b \\
c & d
\end{array}\right]
$$

If $\tau \equiv q^{\nu}$ modulo $p$, then $a=a^{\prime} g^{\nu}, d=d^{\prime} g^{\nu}$ for some $a^{\prime}, d^{\prime} \in C_{H}(u)$. In this case it would be necessary that $b=c=0$. Otherwise, if $\tau$ is not a power of $q$ modulo $p$, then we would have that $a=d=0, c u c^{-1}=u^{r \tau}, b u^{r} b^{-1}=u^{\tau}$. In this case, $r \tau$ would be a power of $q$ modulo $p$, and we would be in the other possibility.

The proof for the normalizer of $S$ is similar. That is, it is clear from the structure of the centralizers that the only subgroup of $S$ that is conjugate to $Q_{1}$ is $\widehat{Q}_{1}=\langle B\rangle$. So anything in the normalizer of $S$ must either normalize both $Q_{1}$ and $\widehat{Q}_{1}$ or interchange them.

Remark 7.5. We should emphasize that the exceptional case (c) in the proposition actually occurs. For example, if $G=\operatorname{SL}(10,2)$ and $p=31$, then $g u g^{-1}=u^{2}$ and we calculate

$$
\left[\begin{array}{cc}
0 & g^{2} \\
1 & 0
\end{array}\right]\left[\begin{array}{cc}
u & 0 \\
0 & u^{15}
\end{array}\right]\left[\begin{array}{cc}
0 & 1 \\
g^{-2} & 0
\end{array}\right]=\left[\begin{array}{cc}
u^{-2} & 0 \\
0 & u
\end{array}\right]=\left[\begin{array}{cc}
u & 0 \\
0 & u^{15}
\end{array}\right]^{-2}
$$

At this point we define functions, $\rho_{0}, \rho_{1}, \rho_{2}: \Gamma_{S, p} \rightarrow \Gamma_{G}$ as in Proposition 5.7. We start with $\rho_{0}(Q)=\left[N_{G}(Q), N_{G}(Q)\right]$ for any nontrivial subgroup $Q$ of $S$. Then we set $\rho_{1}=\widehat{\rho}_{0}$ as constructed in that proposition. So $\rho_{1}(Q)$ is the subgroup of $N_{G}(Q)$ generated by all intersections $N_{G}(Q) \cap \rho_{0}\left(Q^{\prime}\right)$ for $Q^{\prime} \in \Gamma_{S, p}$. Then inductively, let $\rho_{2}=\widehat{\rho}_{1}$ constructed by the same process.

Proposition 7.6. The groups $\rho_{0}(Q)$, for $Q$ in $E$ are given as follows.

(a) $\rho_{0}\left(Q_{1}\right)$ has the form

$$
\rho_{0}\left(Q_{1}\right)=\left\{\left[\begin{array}{ll}
a & 0 \\
0 & b
\end{array}\right] \mid a \in\left\langle w^{q-1}\right\rangle \text { and } b \in \operatorname{SL}(e, q)\right\} .
$$

(b) The subgroup $\rho_{0}\left(Q_{2}\right)$ is generated by $\mathrm{SL}(2, \mathbb{K})$ and elements of the form $X^{[q]} X^{-1}$, for $X \in C_{G}\left(Q_{2}\right)$ where

$$
\left[\begin{array}{ll}
a & b \\
c & d
\end{array}\right]^{[q]}=\left[\begin{array}{ll}
a^{q} & b^{q} \\
c^{q} & d^{q}
\end{array}\right]
$$

(c) The subgroup $\rho_{0}\left(Q_{3}\right)$ is a subgroup of $U$.

(d) $\rho_{0}(S)$ is generated by a subgroup of $U$ and the element $\left[\begin{array}{cc}g & 0 \\ 0 & g^{-1}\end{array}\right]$. 
Proof. This is a direct calculation using Proposition 7.4.

Proposition 7.7. For every nontrivial subgroup $Q$ of $E$ we have that $U \subseteq \rho_{1}(Q)$.

Proof. Let $Q$ be any nontrivial subgroup of $E$ and let $X=\left[\begin{array}{ll}a & 0 \\ 0 & b\end{array}\right]$ be an element of $U$. Then, regarding $a$ and $b$ as elements of $\mathbb{K}$, we must have that $a b=c^{q-1}$ for some $c \in\langle w\rangle$, because the determinant of $X$ is one. That is, we can write

$$
\left[\begin{array}{ll}
a & 0 \\
0 & b
\end{array}\right]=\left[\begin{array}{cc}
a & 0 \\
0 & a^{-1}
\end{array}\right]\left[\begin{array}{cc}
1 & 0 \\
0 & a b
\end{array}\right]
$$

and the latter matrix must have determinant one. But now

$$
\left[\begin{array}{cc}
a & 0 \\
0 & a^{-1}
\end{array}\right] \in \rho_{0}\left(Q_{2}\right) \text { and }\left[\begin{array}{cc}
1 & 0 \\
0 & a b
\end{array}\right] \in \rho_{0}\left(Q_{1}\right)
$$

Because both of these elements are in $U \subseteq N_{G}(Q)$ we have that $X$ is in $\rho_{1}(Q)$.

Next we note some information about a few particular elements.

Lemma 7.8. We have the following

(a)

$$
A_{1}=\left[\begin{array}{cc}
1 & 0 \\
0 & g v^{e-1}
\end{array}\right] \in \rho_{0}\left(Q_{1}\right), \quad \text { and } \quad A_{2}=\left[\begin{array}{cc}
g v^{e-1} & 0 \\
0 & 1
\end{array}\right] \in \rho_{0}\left(\widehat{Q}_{1}\right)
$$

$$
B_{1}=\left[\begin{array}{ll}
g & 0 \\
0 & g
\end{array}\right] \in \rho_{2}\left(Q_{i}\right) \quad \text { for } \quad i=2,3, \quad \text { and } \quad B_{2}=\left[\begin{array}{cc}
0 & 1 \\
-1 & 0
\end{array}\right] \in \rho_{0}\left(Q_{2}\right) \text {; }
$$

$$
C_{1}=\left[\begin{array}{cc}
w & 0 \\
0 & w^{-1}
\end{array}\right] \in \rho_{0}\left(Q_{2}\right), \quad \text { and } \quad C_{2}=\left[\begin{array}{cc}
w^{q-1} & 0 \\
0 & 1
\end{array}\right] \in \rho_{2}\left(Q_{2}\right) .
$$

Proof. The first statement is obvious, because $g v^{e-1} \in \mathrm{SL}(e, q)$. Thus, $A_{2}$ is an element of $\rho_{1}\left(Q_{1}\right)$, and the product $A_{1} A_{2}$ is in $\rho_{2}\left(Q_{2}\right)$ and $\rho_{2}\left(Q_{3}\right)$. Because both $A_{1}$ and $A_{2}$ are in $N_{G}(S)$ we have that $B_{1}$ is in $\rho_{1}(S)$ and hence also in $\rho_{2}\left(Q_{2}\right)$. This proves the first part of the second statement. The second part follows from the fact that $B_{2}$ has determinant equal to one. The first part of (c) is obvious, while the second part follows directly from the fact that $C_{2}$ is the commutator of $A_{2}$ and $C_{1}$, both of which are in $\rho_{1}\left(\widehat{Q}_{1}\right)$, where $\widehat{Q}_{1}=\langle B\rangle$. Hence the commutator is in $\rho_{1}\left(\widehat{Q}_{1}\right) \cap N_{G}\left(Q_{2}\right) \subseteq \rho_{2}\left(Q_{2}\right)$.

Proposition 7.9. For any nontrivial subgroup $Q$ of $E$, we have that $\rho_{2}(Q)=$ $N_{G}(Q)$.

Proof. Recall that $N_{G}\left(Q_{1}\right)$ is generated by $C_{G}\left(Q_{1}\right)=U$ and $A_{2}$. So $\rho_{1}\left(Q_{1}\right)=$ $N_{G}\left(Q_{1}\right)$. Likewise, $\rho_{1}(S)=N_{G}(S)$ because the normalizer of $S$ is generated by $A_{2}$ and $B_{2}$. The normalizer of $Q_{3}$ is generated by $C_{G}\left(Q_{3}\right)=U$ and $B_{1}$. So $\rho_{2}\left(Q_{3}\right)=$ $N_{G}\left(Q_{3}\right)$.

For the normalizer of $Q_{2}$, we note that the element $C_{2}$ when regarded as an element of $\operatorname{GL}(2, \mathbb{K})$ has determinant $w^{q-1}$. As $w$ is a generator of $\mathbb{K}^{\times}$, we get that $C_{G}\left(Q_{2}\right)$ 
is generated by $C_{2}$ and $\operatorname{SL}(2, \mathbb{K})$. Thus, $N_{G}\left(Q_{2}\right)$, which is generated by $C_{G}\left(Q_{2}\right)$ and $B_{1}$, is contained in $\rho_{2}\left(Q_{2}\right)$. This proves the last part.

From all this we can derive the following.

Theorem 7.10. Suppose that $G=\operatorname{SL}(2 e, q)$ for $e>1$, the least integer such that $p$ divides $q^{e}-1$. Then $T T(G)=\{0\}$, and $T(G)=\mathbb{Z}$ is generated by the class of $\Omega(k)$.

Proof. By Theorem 3.4 we need only show that $T T(G)=\{0\}$, which is equivalent to showing that $A(G, S)=\{1\}$ by Theorem 5.2 and using the fact that $T T(S)=\{0\}$. The fact that $A(G, S)=\{1\}$ is proved in Proposition 7.9 and Corollary 5.6.

\section{Endotrivial modules for $\operatorname{SL}(r e, q), 2<r<p$}

We consider the endotrivial modules for the group $G=\operatorname{SL}(n, q)$ where $n=r e$ for $3 \leq r<p$. Here, as before, $e$ is the least integer such that $p$ divides $q^{e}-1$. We assume that $e>1$. Let $\widehat{G}=\operatorname{GL}(n, q)$. Our purpose in this section is to show that $T T(G)$ has order one, that is, the only indecomposable endotrivial module that has trivial Sylow restriction is the trivial module.

To begin, we let $\widehat{L}=\widehat{L}(e, e, \ldots, e) \subseteq \widehat{G}$ be the Levi subgroup of invertible $e \times e$ diagonal block matrices. Let $L=\widehat{L} \cap G$, the corresponding Levi subgroup for $G$. We start with a technical lemma that will be essential to our argument.

Lemma 8.1. Let $N=N_{G}(L)$. With the above hypothesis, we have that $L \subseteq[N, N]$ and $N /[N, N]$ has order 2 .

Proof. The structure of $\widehat{N}=N_{\widehat{G}}(\widehat{L})$ is well known. It has the form

$$
\widehat{N} \cong \widehat{L} \rtimes \mathcal{S}_{r}
$$

where $\mathcal{S}_{r}$ is the symmetric group on $r$ letters. An element of $\widehat{L} \rtimes \mathcal{S}_{r}$ is a pair consisting of a block diagonal matrix with blocks $A_{1}, \ldots, A_{r} \in \mathrm{GL}(e, q)$ and an element $\sigma$ of $\mathcal{S}_{r}$. So we have a map

$$
\varphi: \widehat{N} \longrightarrow\left(\mathbb{F}_{q}^{\times}\right)^{r} \rtimes \mathcal{S}_{r}
$$

where $\varphi\left(\left(A_{1}, \ldots, A_{r}, \sigma\right)\right)=\left(\operatorname{Det}\left(A_{1}\right), \ldots, \operatorname{Det}\left(A_{r}\right), \sigma\right)$. One can verify that $\varphi$ is a homomorphism. Note that the kernel of $\varphi$ is $\operatorname{SL}(e, q)^{\times r}$ which is a perfect group and hence is contained in the commutator subgroup $[N, N]$.

Now note that $\left(\mathbb{F}_{q}^{\times}\right)^{r} \rtimes \mathcal{S}_{r}$ is naturally isomorphic to $N_{G L(r, q)}(T)$, the normalizer in $\mathrm{GL}(r, q)$ of the torus $T$ of invertible diagonal matrices. From Lemma 4.2, we know that $T \subseteq\left[N_{G L(r, q)}(T), N_{G L(r, q)}(T)\right]$. It is an easy check that $\varphi(\widehat{N})=N_{G L(r, q)}(T) \cap$ $\mathrm{SL}(r, q)$. Hence, $N /[N, N] \cong \mathcal{S}_{r} /\left[\mathcal{S}_{r}, \mathcal{S}_{r}\right]$ has order 2 .

Lemma 8.2. Every indecomposable $k L$-module with trivial Sylow restriction has dimension one.

Proof. Let $U=\mathrm{GL}(e, q)$, and let $S_{1}$ be a Sylow $p$-subgroup of $U$. Let $\widehat{H}=N_{U}\left(S_{1}\right) \times$ $U^{\times(r-1)} \subseteq \widehat{L}$ be the subgroup of diagonal $e \times e$ blocks in $\operatorname{GL}(n, q)$, the first of which is in $N_{U}\left(S_{1}\right)$ and the last $r-1$ being in $U$. Let $H=\widehat{H} \cap G$. Note that $H$ has a nontrivial normal $p$-subgroup. Hence, every $k H$-module with trivial Sylow 
restriction has dimension one. Note that $\widehat{L} \cong U^{\times r}$ and we see that every left coset of $H$ in $L$ is represented by an element having the block form $X \times \operatorname{Id}_{U}^{\times(r-1)}$, for some $X \in U$ of determinant one, where $\operatorname{Id}_{U}$ is the identity element for $U$. Every one of these elements centralizes a nontrivial $p$-subgroup of $H$, namely a subgroup of the form $S_{1} \times \operatorname{Id}_{U}^{\times(r-1)}$. The lemma now follows from Proposition 2.6.

The following two lemmas deal with situations when $M$ has trivial Sylow restriction.

Lemma 8.3. Let $M$ is a $k G$-module with trivial Sylow restriction (i.e., $M_{\downarrow S} \cong$ $k \oplus$ (proj).) The restriction of $M$ to $L$ has the form

$$
M_{\downarrow L} \cong k \oplus(\text { proj }) \text {. }
$$

Proof. Let $N=N_{G}(L)$. Write $M_{\downarrow N}=V \oplus$ (proj) where $V$ is an indecomposable $k N$-module with trivial Sylow restriction. We must have that $V_{\downarrow L} \cong X \oplus$ (proj) where $X$ has dimension one, by Lemma 8.2. Because $L$ is normal in $N$, and $V$ is a direct summand of $X^{\uparrow N}$, it follows from Clifford theory and the fact that $V$ is endotrivial that $V$ has dimension one. Thus, $X \cong k$, since $L$ is in the commutator subgroup of $N$.

Lemma 8.4. Let $M$ be a $k G$-module with trivial Sylow restriction, and let $\mathcal{L}=$ $L((r-1) e, e)$ be the Levi subgroup that is the intersection of $G$ with $\mathrm{GL}((r-1) e, q) \times$ $\operatorname{GL}(e, q) \subseteq \operatorname{GL}(n, q)$. Then $M_{\downarrow \mathcal{L}} \cong k \oplus$ (proj). Hence, $M$ is a direct summand of $k_{\mathcal{L}}^{\uparrow G}$.

Proof. This is another argument using the Mackey formula. Let $M_{\downarrow \mathcal{L}}=V \oplus$ (proj), where $V$ is indecomposable. Then by the previous lemma, $V$ is a direct summand of $k_{L}^{\uparrow \mathcal{L}}$. However, all of the $L-L$ double cosets in $\mathcal{L}$ can be chosen to be in the factor $\operatorname{GL}((r-1) e, q) \cap G$ which centralizes a nontrivial $p$-subgroup in the factor $\operatorname{GL}(e, q) \cap G$. Consequently, $\left(k_{L}^{\uparrow \mathcal{L}}\right)_{\downarrow L}$ can have no projective summands by the Mackey formula. Thus, $V \cong k_{\mathcal{L}}$.

Lemma 8.5. The modules $k_{L}^{\uparrow G}$ and $k_{\widehat{L}}^{\uparrow \widehat{G}}$ each have at most two indecomposable endotrivial direct summands. In each case, one of these summands is the trivial module $k$.

Proof. First notice that, because $L \subseteq \widehat{L}$, we have that $\left(k_{\widehat{L}}^{\uparrow \widehat{G}}\right)_{\downarrow G}$ is isomorphic to a direct summand of $k_{L}^{\uparrow G}$. So the result for $k_{\widehat{L}}^{\uparrow \widehat{G}}$ follows from the result for $k_{L}^{\uparrow G}$. Because $N=N_{G}(L)$ contains the normalizer of the Sylow subgroup $S$, we have that any endotrivial $k G$-module is the Green correspondent of an endotrivial $k N$-module, By transitivity of induction, $k_{L}^{\uparrow G}=\left(k_{L}^{\uparrow N}\right)^{\uparrow G}$, and there are no more endotrivial $k G$-direct summands of $k_{L}^{\uparrow G}$ than there are endotrivial $k N$-direct summands of $k_{L}^{\uparrow N} \cong k \mathcal{S}_{r}$. The lemma is a consequence of the fact that there are exactly two one dimensional direct summands of $k_{L}^{\uparrow G}$, namely $k$ and the sign module.

From this point we use the notation. Let $\widehat{\mathcal{L}}=\widehat{L}((r-1) e, e)=\operatorname{GL}((r-1) e, q) \times$ $\operatorname{GL}(e, q)$ and let $\mathcal{L}=\widehat{\mathcal{L}} \cap G$. 
Lemma 8.6. With the above notation, we have that

$$
k_{\mathcal{L}}^{\uparrow G} \cong\left(k_{\widehat{\mathcal{L}}}^{\uparrow \widehat{G}}\right)_{\downarrow G}
$$

Proof. From the Mackey formula, we have that

$$
\left(k_{\widehat{\mathcal{L}}}^{\uparrow \widehat{G}}\right)_{\downarrow G}=\bigoplus_{x \in[G \backslash \widehat{G} / \widehat{\mathcal{L}}]} x \otimes k_{G \cap \widehat{\mathcal{L}}}^{\uparrow G} \cong k_{\mathcal{L}}^{\uparrow G}
$$

since $G \widehat{\mathcal{L}}=\widehat{G}$ and there is only one $G-\widehat{\mathcal{L}}$ double coset which is represented by $x=1$.

Now we invoke the theory of Young modules. We have that

$$
k_{\widehat{\mathcal{L}}}^{\uparrow \widehat{G}} \cong Y_{[(r-1) e, e]} \oplus \bigoplus_{j=1}^{e-1}\left(Y_{[n-j, j]}\right)^{a_{j}} \oplus Y_{[n]},
$$

for some multiplicities $a_{j}$. Note that $Y_{[n]}=k$ has multiplicity 1 by Frobenius reciprocity. Hence we only need to deal with the other summands.

Lemma 8.7. If $Y=\left(Y_{[(r-1) e, e]}\right)_{\downarrow G}$ has an endotrivial direct summand, then it has only one. In that case $Y$ is indecomposable and $Y_{[(r-1) e, e]}$ is endotrivial as a $k \widehat{G}$ module.

Proof. The fact that there is at most one endotrivial direct summand of $Y$ is a consequence of Lemma 8.5. By Clifford theory, the module $Y$ is a direct sum of conjugate $k G$-modules. If one of the summands is an endotrivial module then they all must be endotrivial. Since, by Lemma 8.5 there can be only one, $Y$ must be indecomposable and endotrivial. This means that it is also endotrivial as a $k \widehat{G}$ module.

Let $A$ be an invertible matrix of order $p$ in $\operatorname{SL}(e, q)$, and let $X$ be the diagonal block matrix in $\operatorname{SL}(r e, q)$ with $r$ diagonal blocks, each equal to $A$. We assume that the Sylow $p$-subgroup $S$ is chosen so that $X \in S$. Let $U=\langle X\rangle$ be the subgroup generated by $X$ in $G$.

Lemma 8.8. For all $1 \leq j<e$, the restriction of the Young module $Y_{[n-j, j]}$ to $U$ is a free module. Hence, $Y_{[n-j, j]}$ and its restriction to $G$ have no endotrivial direct summands.

Proof. Let $\widetilde{L}=\widehat{L}(n-j, j) \cong \mathrm{GL}(n-j, q) \times \mathrm{GL}(j, q)$. We claim that for any $x \in \widehat{G}$, $U \cap x \widetilde{L} x^{-1}=\{1\}$. Otherwise the matrix $x^{-1} X x$ would have the form of diagonal blocks $X_{1} \in \mathrm{GL}(n-j, q)$ and $X_{2} \in \operatorname{GL}(j, q)$. However, the order of $\operatorname{GL}(j, q)$ is not divisible by $p$ and hence, $X_{2}$ is the identity matrix. This is a contradiction because $X$ was chosen to have no eigenvalues equal to 1 . This proves the claim. The lemma now follows by an easy argument using the Mackey formula.

Lemma 8.9. The restriction of the Young module $Y=Y_{[(r-1) e, e]}$ to $U$ has at least two trivial direct summands. That is, $Y_{\downarrow U} \cong k^{a} \oplus k U^{b}$, for some multiplicities a and $b$ where $a>1$. 
Proof. Consider the Mackey formula

$$
\left(k_{\widehat{\mathcal{L}}}^{\uparrow \widehat{G}}\right)_{\downarrow U} \cong \sum_{x \in[U \backslash \widehat{G} / \widehat{\mathcal{L}}]} x \otimes k_{U \cap \widehat{\mathcal{L}}^{x}}^{\uparrow U}
$$

Clearly, the multiplicity of $k_{U}$ as a direct summand is precisely the number of $U$ - $\widehat{\mathcal{L}}$ double cosets represented by elements in $N_{G}(U)$. Note that if $x \in N_{G}(U)$ then $U x \widehat{\mathcal{L}}=x \widehat{\mathcal{L}}$. Now $N_{G}(U)$ contains a subgroup $\Sigma \cong \mathcal{S}_{r}$. This subgroup is in the normalizer of the Levi subgroup $\widehat{L}(e, e, \ldots, e)$ of block diagonal $e \times e$ invertible matrices, where the conjugation action of $\Sigma$ permutes the blocks. The intersection of $\Sigma$ with $\widehat{\mathcal{L}}$ is isomorphic to $\mathcal{S}_{r-1}$. Thus we have that $\widehat{\mathcal{L}}$ has at least $|\Sigma| /|\Sigma \cap \widehat{\mathcal{L}}|=r$ distinct left cosets represented by element of $N_{G}(U)$. Hence, the multiplicity of $k_{U}$ as a direct summand of $\left(k_{\widehat{\mathcal{L}}}^{\uparrow \widehat{G}}\right)_{\downarrow U}$ is at least $r \geq 3$.

By Lemma 8.8, the multiplicity of $k_{U}$ as a direct summand of $\left(Y_{[n-j, j]}\right)_{\downarrow U}$ is zero for $1 \leq j<e$. The multiplicity $k_{U}$ in $\left(Y_{[n]}\right)_{\downarrow U} \cong k$ is exactly one. So the lemma follows from the decomposition of $k_{\widehat{\mathcal{L}}}^{\uparrow \widehat{G}}$ as a sum of Young modules.

The above results are sufficient to prove the main theorem of this section.

Theorem 8.10. Suppose that $n=$ re for $e>1$ and $r \geq 2$, and that $G=\operatorname{SL}(n, q)$. Then $T T(G)=\{0\}$.

Proof. First, for $r=2$ the claim is proved in Theorem 7.10. Now suppose that $r>2$ and that $M$ is an indecomposable endotrivial $k G$-module with trivial Sylow restriction and $M \neq k$. Then by Lemma $8.4, M$ is a direct summand of $k_{\mathcal{L}}^{\uparrow G}$. Then by Lemmas 8.6, 8.7 and 8.8, $M$ is isomorphic to the restriction of $Y_{[(r-1) e, e]}$ to $G$ and $Y_{[(r-1) e, e]}$ must be an endotrivial $k \widehat{G}$-module. However, by Lemma 8.9 , this is not the case.

\section{Endotrivial modules for $\operatorname{SL}(r e+f, q), 2 \leq r<p, 1 \leq f<e$}

In this section, we present the final case of the endotrivial modules of $\operatorname{SL}(n, q)$ under the assumption that $S$ is abelian, namely, the case in which $n=r e+f$ with $r<p$ and $f \geq 1$. In the proof we again use Balmer's method for computing the kernel of the restriction map. Indeed, we use the method twice in different contexts. On one occasion, we show that $A(G, H)=\{1\}$ where $H$ is the commutator subgroup of the maximal parabolic subgroup $P(n-1,1)$. The proof is by induction on $f$, the result being known for the case that $f=0$ by the main theorem of the last section. The theorem that we prove is slightly more general than what has been stated above. We hope this generality will be useful in later work.

Because we assume that $f>1$, the power of $p$ dividing $|\mathrm{SL}(n-1, q)|$ is the same as that dividing $|\operatorname{SL}(n, q)|$, so that the Levi subgroup $L=L(n-1,1)$ contains a Sylow $p$-subgroup of $G$. For notation, let $P=P(n-1,1)$, the maximal parabolic subgroup of $G=\operatorname{SL}(n, q)$ consisting of upper block triangular invertible matrices with diagonal blocks of size $(n-1) \times(n-1)$ and $1 \times 1$, having the product of their 
determinants equal to 1 . So an element of $P$ has the form

$$
X \quad:=\left[\begin{array}{cc}
A & v \\
0 & \zeta
\end{array}\right]
$$

where $A \in \operatorname{GL}(n-1, q), \zeta \in k^{\times}$and $v \in \mathbb{V}$, the natural module for $\operatorname{GL}(n-1, q)$ (the $k$-vector space of dimension $n-1$ with elements written as column vectors). Also, $\operatorname{Det}(A)=\zeta^{-1}$. Let $H=[P, P]$, the commutator subgroup of $P$. It is not difficult to prove that $H$ consists of all elements of the above form $X$ with the additional stipulations that $A \in \mathrm{SL}(n-1, q)$ and $\zeta=1$. The element $v$ can still be any element of $\mathbb{V}$.

Let $V \subseteq H$ be the set of all elements having the above form with $A=I_{n-1}$ the identity matrix and $\zeta=1$. So the map $\varphi: \mathbb{V} \rightarrow V$ that sends $v \in \mathbb{V}$ to $\left[\begin{array}{cc}I_{n-1} & v \\ 0 & 1\end{array}\right]$ is an isomorphism of abelian groups. Indeed, if $\vartheta: \mathrm{SL}(n-1, q) \rightarrow H$ is the injection that sends $A$ to $\left[\begin{array}{ll}A & 0 \\ 0 & 1\end{array}\right]$, then we have that $\varphi(A v)=\vartheta(A) \varphi(v) \vartheta(A)^{-1}$. This fact is very useful in one of the proofs that follow. Finally, let $L \cong \operatorname{SL}(n-1, q)$ denote the image of $\vartheta$. Thus, $V$ is a normal subgroup of $H$ with quotient $H / V \cong L$.

Lemma 9.1. There are exactly two $L$-L-double cosets in $H$, and they are represented by 1 and the element $w=\varphi((0,0, \ldots, 1))$.

Proof. Let $\hat{w}=(0, \ldots, 0,1) \in \mathbb{V}$ a preimage of $w$ under $\varphi$. Because $\mathbb{V}$ is an irreducible module and $\operatorname{SL}(n-1, q)$ acts transitively on $\mathbb{V}$, for any nonzero $v \in V$ with preimage $\hat{v}$ in $\mathbb{V}$, we have that there is some $A$ in $\operatorname{SL}(n-1, q)$ such that $A \hat{v}=\hat{w}$ and $\vartheta(A) v=w$. On double cosets this means that $L v L=L w L$ for all $v \neq 1$ in $V$. Since $H=V L$, we have proved the lemma.

Proposition 9.2. The restriction map $T(H) \rightarrow T(L)$ is injective. Hence, if $T T(L)=$ $\{0\}$, then $T T(H)=\{0\}$.

Proof. We have an element of order $p$ of the form $U=\left[\begin{array}{cc}u & 0 \\ 0 & I_{n-e}\end{array}\right]$ in $H$, where $u$ is an element of order $p$ in $\operatorname{SL}(e, q)$. The centralizer contains the subgroup $J$ of all matrices of the form $\left[\begin{array}{cc}I_{e} & 0 \\ 0 & A\end{array}\right]$ for $A$ in $\operatorname{SL}(n-e, k)$. The element $w$ of the previous lemma is in $J$ and in the commutator subgroup of the centralizer of $U$. So by Lemma 9.1 and Proposition 5.4, the group $A(H, L)$ of weak $L$-homomorphisms is trivial. So by Theorem 5.2, the restriction $T(H) \rightarrow T(L)$ is injective.

Lemma 9.3. The left and right cosets of $H$ in $P$ are represented by elements of the form

for all $\zeta \in \mathbb{F}_{q}^{\times}$.

$$
a_{\zeta}=\left[\begin{array}{cc}
I_{n-2} & 0 \\
0 & Y
\end{array}\right] \quad \text { where } \quad Y=\left[\begin{array}{cc}
\zeta & 0 \\
0 & \zeta^{-1}
\end{array}\right]
$$

Proof. This is a direct calculation.

Lemma 9.4. There are exactly two P-P double cosets in $G$, that are represented by the elements 1 and

$$
b=\left[\begin{array}{cc}
I_{n-2} & 0 \\
0 & A
\end{array}\right] \quad \text { where } \quad A=\left[\begin{array}{cc}
0 & 1 \\
-1 & 0
\end{array}\right],
$$


Proof. We obtain a complete set of double coset representatives from [13, Prop. 2.8.1(iii)] as follows. Let $\Delta=\left\{\alpha_{1}, \alpha_{2}, \ldots, \alpha_{n-1}\right\}$ be the simple roots. Set $P_{J}=P$ where $\Delta_{J}=\left\{\alpha_{1}, \alpha_{2}, \ldots, \alpha_{n-2}\right\} \subseteq \Delta$. Let $D_{J}=\left\{w \in W: w\left(\Delta_{J}\right) \subseteq \Phi^{+}\right\}\left(\Phi^{+}\right.$is the set of positive roots). Let $s_{j}=s_{\alpha_{j}}$ for $j=1,2, \ldots, n-1$. In this case

$$
D_{J}=\left\{1, s_{n-1}, s_{n-2} s_{n-1}, \ldots, s_{1} s_{2} \cdots s_{n-1}\right\} .
$$

Then a set of double coset representatives are given by $D_{J} \cap D_{J}^{-1}=\left\{1, s_{n-1}\right\}$ (cf. $\left[13,2.7\right.$ Definition]). The matrix $b$ represents $s_{n-1} \in W$.

Lemma 9.5. Every $H-H$ double coset in $G$ is represented by an element of the form

$$
X=\left[\begin{array}{cc}
I_{n-2} & 0 \\
0 & W
\end{array}\right] \quad \text { where } \quad W \in \operatorname{SL}(2, q) .
$$

Any such $X$ is in the commutator subgroup of the subgroup $U$ in the proof of Proposition 9.2

Proof. Every element of $x \in G$ has either the form $x=u$ or $x=u b v$ for $u, v \in P$ by Lemma 9.4. So by Lemma 9.3 we can write $u=h a_{\zeta}$ and $v=a_{\eta} h^{\prime}$ for some $h, h^{\prime} \in H$ and some $\zeta, \eta \in \mathbb{F}_{q}^{\times}$. Thus the double coset representatives can be taken to have the form $a_{\zeta}$ for some $\zeta$, or $a_{\zeta} b a_{\eta}$ for some $\zeta$ and some $\eta$. This proves the lemma as the last statement is obvious.

At this point we can prove the main theorem of the section. We regard $\operatorname{SL}(n-1, q)$ as the subgroup of $\operatorname{SL}(n, q)$ consisting of block diagonal matrices of sizes $(n-1) \times$ $(n-1)$ and $1 \times 1$. This is exactly as $L \subseteq G$ in the notation above.

Theorem 9.6. Suppose that $\operatorname{SL}(n-1, q)$ contains a Sylow $p$-subgroup of $G$ and the Sylow p-subgroup has p-rank at least two. Then the group $A(\operatorname{SL}(n, q), \operatorname{SL}(n-1, q))$ of weak $\mathrm{SL}(n-1, q)$-homomorphisms of $\mathrm{SL}(n, q)$ is trivial, and so the restriction map $T(\mathrm{SL}(n, q)) \rightarrow T(\mathrm{SL}(n-1, q))$ is injective.

Proof. The hypothesis of the Sylow $p$-subgroup of $G$ assures us that $n>2 e$. Suppose that $H=\mathrm{SL}(n-1, q) \subseteq G=\mathrm{SL}(n, q)$. Then we argue as in the proof of Proposition 9.2 that by Lemma 9.5, a complete set of representatives of $H-H$ double cosets can be chosen in the commutator subgroup of the centralizer of a nontrivial $p$-subgroup. Thus by Proposition 5.4 and Theorem 5.2, we conclude that $A(G, H)=\{1\}$ and the restriction map $T(G) \rightarrow T(H)$ is injective. So the composition of restriction maps $T(G) \rightarrow T(L)$ is injective by Proposition 9.2.

Corollary 9.7. Suppose that $n=r e+f$ for $2 \leq r<p$ and $0 \leq f<e$. Then $T T(\operatorname{SL}(n, q))=\{0\}$.

Proof. According to Theorem 8.10 we have $T T(\operatorname{SL}(r e, q))=\{0\}$. Now one can apply induction on $f$ using Theorem 9.6 to complete the proof of the corollary.

\section{Proofs of Theorems 1.1 And 1.2}

In this section we prove the theorems of the introduction. The proof in the case that the Sylow $p$-subgroup is cyclic is a direct calculation based on known results. The proof in the case that the Sylow $p$-subgroup is abelian and has $p$-rank at least 
2 is a compilation of results from prior sections. All of this is extended to finite groups of Lie type with underlying root system of type $A_{n}$.

Proof of Theorem 1.1. We have proved that $T(S L(n, q))=\mathbb{Z}$ whenever the Sylow $p$-subgroup is abelian and has $p$-rank at least 2 in Theorems 3.4, 7.10, 8.10 and 9.7. If $\mathrm{SL}(n, q) \subseteq G \subseteq \mathrm{GL}(n, q)$, then a proof that $T(G) \cong \mathbb{Z} \oplus X(G)$ can be constructed using Clifford theory. That is, since any indecomposable endotrivial $k G$-module, restricts to a sum of conjugate $k \mathrm{SL}(n, q)$-modules, the restriction must have only one summand and must have dimension one if the endotrivial module has trivial Sylow restriction.

If $Z \subseteq Z(G)$, then we note that any endotrivial $k(G / Z)$-module must inflate to an endotrivial $k G$-module.

Proof of Theorem 1.2. Observe that if $p$ divides $d$ or if $p=2$, then the assumption that a Sylow $p$-subgroup of $G$ is cyclic, requires that $n=1$. So in this case $G \cong D$ is abelian and the structure of $T(G / Z)$ is easily computed.

Recall from Theorem 2.7 that $T(G / Z) \cong T(\widetilde{N})$ where $\widetilde{N}=N_{G / Z}(\widetilde{S})$. Here, $\widetilde{S}$ is the unique subgroup of order $p$ in a Sylow $p$-subgroup of $G / Z$. In this case, the normalizer of $\widetilde{S}$ coincides with the normalizer of $\widehat{S}$, the Sylow $p$-subgroup of $G / Z$. Note that $\widehat{S} \cong Z S / Z$ and its normalizer is $\widehat{N} \cong N / Z$. The displayed sequence (1) follows from the sequence in Theorem 2.7 and the fact that $X(\widehat{N}) \cong \widehat{N} /[\widehat{N}, \widehat{N}]$ where $\widehat{S} \subseteq[\widehat{N}, \widehat{N}]$ and

$$
\widehat{N} /[\widehat{N}, \widehat{N}] \cong(N / Z) /[N / Z, N / Z] \cong(N / Z) /(Z[N, N] / Z) \cong N /(Z[N, N]) .
$$

Now assume that $p>2$ divides $q-1$, but not $d$. Then $n=2$, and $N$ is generated by elements

$$
x=\left[\begin{array}{cc}
0 & 1 \\
-1 & 0
\end{array}\right], \quad u_{a}=\left[\begin{array}{ll}
a & 0 \\
0 & 1
\end{array}\right], \quad \text { and } \quad v_{b}=\left[\begin{array}{cc}
b & 0 \\
0 & b^{-1}
\end{array}\right]
$$

for $a \in D$, and $b \in \mathbb{F}_{q}^{\times}$. The Sylow $p$-subgroup is generated by $u_{\zeta}$ where $\zeta \in \mathbb{F}_{q}^{\times}$ generates a Sylow $p$-subgroup of $\mathbb{F}_{q}^{\times}$.

Let $T$ denote the torus in $\operatorname{SL}(n, q)$ that consists of all $v_{b}$ for $b \in \mathbb{F}_{q}^{\times}$. It is easily seen that the commutator subgroup $[N, N]$ of $N$ is generated by all $x^{-1} u_{a^{-1}} x u_{a}=v_{a}$ and $x^{-1} v_{b^{-1}} x v_{b}=v_{b^{2}}$. It follows that $[N, N]=T$ if and only if $D$ contains a generator for the Sylow 2-subgroup of $\mathbb{F}_{q}^{\times}$. This happens if and only if 2 does not divide $(q-1) / d$. Otherwise, the index of $[N, N]$ in $T$ is 2 .

In the first case when $[N, N]=T$, we have that $N /[N, N] \cong D \times C_{2}$ where the factors are generated by the classes (modulo $[N, N]$ ) of $u_{a}$ for $a$ a generator of $D$ and $x$. Then $T(G) \cong T(N) \cong \mathbb{Z} / d \mathbb{Z} \oplus \mathbb{Z} / 4 \mathbb{Z}$ because the element $x$ acts by inverting the elements of $S$ and hence the class of $\Omega(k)$ in $T(G)$ has order 4 . That is, $\Omega^{2}(k)$ is a $k G$-module of dimension one on which $x$ acts by multiplication by -1 .

In the second case, when the index of $[N, N]$ in $T$ is 2 , we have that $N /[N, N] \cong$ $D \times C_{2} \times C_{2}$ where the factors are generated by the classes of $u_{a}$, for a generator $a$ of $D, x$, and $v_{b}$, for a generator $b$ of the Sylow 2-subgroup of $T$. Then

$$
T(G) \cong T(N) \cong \mathbb{Z} / d \mathbb{Z} \oplus b Z / 4 \mathbb{Z} \oplus \mathbb{Z} / 2 \mathbb{Z},
$$


for the same reason as above.

We invoke the notation in Section 6. Suppose that $e>1$ and assume first that $f=0$. By Lemma 6.2, parts (a) and (c), we have that $C_{G}(S)=\left\langle w^{m}\right\rangle=G \cap C_{\widehat{G}}(S)$ for $\widehat{G}=\operatorname{GL}(e, q)$ and $m=(q-1) / d$. Thus by part (b), $N$ is generated by $w^{m}$ and $g$. We can see that $[N, N]$ is generated by $g w^{m} g^{-1} w^{-m}=w^{m(q-1)}$. So the index of $[N, N]$ in $C_{G}(S)$ is $\ell=\operatorname{gcd}\left(m(q-1), q^{e}-1\right) / m$. It follows that $N /[N, N] \cong C_{\ell} \times C_{e}$, and that $T(G)$ has the asserted form.

Finally suppose that $e>1$ and $f>0$. Let $\theta: \operatorname{GL}(e, q) \rightarrow \operatorname{SL}(n, q) \subseteq G$ be the homomorphism that takes an element $x$ to the block matrix with $x$ in the upper left corner. The lower right corner of $\theta(x)$ is the diagonal $f \times f$ matrix with $\operatorname{Det}(x)^{-1}$ in the upper left corner, the other diagonal entries being equal to 1. The normalizer $N_{G}(S)$ consists of all elements of the form $\left[\begin{array}{cc}A & 0 \\ 0 & B\end{array}\right]$ such that $A \in N_{\mathrm{GL}(e, q)}(\langle u\rangle), B \in \mathrm{GL}(f, q)$ and $\operatorname{Det}(A) \operatorname{Det}(B) \in D=\operatorname{Det}(G)$. Then, in almost all cases, we see that $[N, N]$ is generated by $\theta\left(w^{q-1}\right)$ and all elements (as above) with $A=I_{e}$ and $B \in \operatorname{SL}(f, q)$. The exception to the above occurs when $f=2$ and $q=2$, because in that case the commutator subgroup of $\operatorname{GL}(f, q)$ is not equal to $\operatorname{SL}(f, q)$, but is a subgroup of index 2. As a consequence we have (in all but the noted exceptional case) that $N /[N, N] \cong C_{e} \times C_{q-1} \times C_{d}$, where the first two factors are generated by the classes modulo $[N, N]$ of $\theta(g)$ and $\theta(w)$. The third factor is generated by the class of an element given by the diagonal matrix that has 1 in every diagonal entry except the $(e+1) \times(e+1)$ entry which is a generator for the group $D \subseteq \mathbb{F}_{q}^{\times}$. Then $T(G)$ has the asserted form.

\section{REFERENCES}

[1] J. Alperin, A construction of endo-permutation modules, J. Group Theory, 4, (2001), $3-10$.

[2] A. Adem, R. Milgram, Cohomology of finite groups, Grundlehren der Mathematischen Wissenschaften, 309. Springer-Verlag, Berlin, 1994.

[3] P. Balmer, Modular representations of finite groups with trivial restriction to Sylow subgroups, J. European Math. Soc., 15, (2013), 2061-2079.

[4] P. Balmer, Stacks of group representations, J. European Mathematical Society, (to appear).

[5] W. Bosma, J.Cannon, Handbook on Magma Functions, Sydney University, 1996.

[6] J. Carlson, Constructing Endotrivial Modules, J. Pure and Appl. Algebra, 206, (2006), $83-110$.

[7] J. Carlson, D. Hemmer and N. Mazza, The group of endotrivial modules for the symmetric and alternating groups, Proc. Edinburgh Math. Soc., 53, (2010), 83-95.

[8] J. Carlson, N. Mazza and D. Nakano, Endotrivial modules for finite groups of Lie type, J. Reine Angew. Math., 595, (2006), 93-120.

[9] J. Carlson, N. Mazza and D. Nakano, Endotrivial modules for the symmetric and alternating groups, Proc. Edinburgh Math. Soc., 52, (2009), 45-66.

[10] J. Carlson, N. Mazza and J. Thévenaz, Endotrivial modules for $p$-solvable groups, Trans. Amer. Math. Soc., 363, (2011), no. 9, 4979-4996.

[11] J. Carlson and J. Thévenaz, The classification of endo-trivial modules, Invent. Math. 158 (2004), no. 2, 389-411.

[12] J. Carlson and J. Thévenaz, The classification of torsion endotrivial modules, Ann. of Math., 165, (2005), 823-883. 
[13] R. W. Carter, Finite Groups of Lie Type: Conjugacy Classes and Complex Characters, John Wiley and Sons, 1985.

[14] E. C. Dade, Endo-permutation modules over p-groups, I, Ann. Math., 107, (1978), 459494.

[15] E. C. Dade, Endo-permutation modules over p-groups II, Ann. Math., 108, (1978), $317-$ 346.

[16] K. Erdmann, S. Schroll, On Young modules of general linear groups, J. Algebra, 310, (2007), no. $1,434-451$.

[17] D. Gorenstein, Finite Groups, Harper \& Roe, New York, 1968.

[18] D. Gorenstein, R. Lyons, R. Solomon, The Classification of the Finite Simple Groups, Volume 40, Number 3, AMS, 1998.

[19] C. Lassueur, G. Malle, E. Schulte, Simple endotrivial modules for quasi-simple groups, J. Reine Angew. Math., to appear.

[20] N. Mazza and J. Thévenaz, Endotrivial modules in the cyclic case, Arch. Math., 89, (2007), 497-503.

[21] G. Navarro and G. Robinson, On endo-trivial modules for p-solvable groups, Math. Z., 270, (2012), no. 3-4, 983-987.

[22] G. Robinson, On simple endotrivial modules, Bull. Lond. Math. Soc., 43, (2011), no. 4, $712-716$.

[23] A. Weir, Sylow p-subgroups of the classical groups over finite fields with characteristic prime to p, Proc. Amer. Math. Soc., 6, (1955), 529-533.

Department of Mathematics, University of Georgia, Athens, GA 30602, USA

E-mail address: jfc@math.uga.edu

Department of Mathematics, University of Lancaster

E-mail address: nmazza@math.uga.edu

Department of Mathematics, University of Georgia, Athens, GA 30602, USA

E-mail address: nakano@math.uga.edu 